\title{
Inclined cross-stream stereo particle image velocimetry measurements in turbulent boundary layers
}

\author{
By N. HUTCHINS, W. T. HAMBLETON AND IVAN MARUSIC \\ Aerospace Engineering and Mechanics, University of Minnesota, 107 Akerman Hall, \\ 110 Union St SE, Minneapolis, MN 55455, USA
}

(Received 1 June 2004 and in revised form 19 April 2005)

This work can be viewed as a reprise of Head \& Bandyopadhyay's (J. Fluid Mech. vol. 107,1981, p. 297) original boundary-layer visualization study although in this instance we make use of stereo particle image velocimetry (PIV), techniques to obtain a quantitative view of the turbulent structure. By arranging the laser light-sheet and image plane of a stereo PIV system in inclined spanwise/wall-normal planes (inclined at both $45^{\circ}$ and $135^{\circ}$ to the streamwise axis) a unique quantitative view of the turbulent boundary layer is obtained. Experiments are repeated across a range of Reynolds numbers, $R e_{\tau} \approx 690-2800$. Despite numerous experimental challenges (due to the large out-of-plane velocity components), mean flow and Reynolds stress profiles indicate that the salient features of the turbulent flow have been well resolved. The data are analysed with specific attention to a proposed hairpin eddy model. In-plane two-dimensional swirl is used to identify vortical eddy structures piercing the inclined planes. The vast majority of this activity occurs in the $135^{\circ}$ plane, indicating an inclined eddy structure, and Biot-Savart law calculations are carried out to aid in the discussion. Conditional averaging and linear stochastic estimation results also support the presence of inclined eddies, arranged about low-speed regions. In the $135^{\circ}$ plane, instantaneous swirl patterns exhibit a predisposition for counter-rotating vortex pairs (arranged with an ejection at their confluence). Such arrangements are consistent with the hairpin packet model. Correlation and scaling results show outer-scaling to be the correct way to quantify the characteristic spanwise length scale across the $\log$ and wake regions of the boundary layers (for the range of Reynolds numbers tested). A closer investigation of two-point velocity correlation contours indicates the occurrence of a distinct two-regime behaviour, in which contours (and hence streamwise velocity fluctuations) either appear to be 'attached' to the buffer region, or 'detaching' from it. The demarcation between these two regimes is found to scale well with outer variables. The results are consistent with a coherent structure that becomes increasingly uncoupled (or decorrelated) from the wall as it grows beyond the logarithmic region, providing additional support for a wall-wake description of turbulent boundary layers.

\section{Introduction}

At practical Reynolds numbers, turbulent boundary layers are an unavoidable feature of most fluid/solid-body interactions. Despite decades of work, it is fair to conclude that an incomplete understanding of these flows has long handicapped the design of numerous fluid-mechanical systems; from duct or pipe flows, to aircraft 
and hydrodynamic vehicles. The current goal behind a great deal of turbulence research is to understand better the underlying physics of wall-bounded fluid flows, with the ultimate aim of producing improved and workable prediction and control strategies.

There has been a gradual discovery over the past six or so decades that turbulent boundary layers possess recurrent quantifiable features and flow topologies, collectively referred to as coherent structures. There are many excellent review papers that describe this discovery process and provide full lists of references. Kline \& Robinson (1988) and Robinson (1991) provide summaries of the research as it stood a decade ago (the latter references over 150 papers); a more recent review is given by Panton (2001). These coherent structures have been shown to play an important role in turbulence production, being major contributors to time-averaged turbulence statistics, including skin friction. The spatio-temporal information associated with such recurrent features had beforehand been largely absent from a purely statistical description of turbulent boundary layers, and has obvious applications to control and prediction.

Whilst the exact form of the near-wall quasi-streamwise structure continues to generate controversy, there is a general emergent consensus on the existence of hairpin-shaped vortical structures in the $\log$ and wake regions of the turbulent boundary layer. Indeed, at such distances from the wall, these models have proved remarkably persuasive at describing the salient features and mean flow statistics. Theodorsen (1952) and Townsend (1956) are universally quoted as not only accepting the existence of, but also proposing a surprisingly convincing model for the coherent turbulent structure. Theodorsen, in particular, invoked a model of inclined hairpin vortical structures. Experimental confirmation of this model began to gather pace in the 1960s and 1970s. From early flow-visualization experiments, Kline et al. (1967) and Offen \& Kline (1975) proposed an oft-revisited model of streak break-up due to a lifted and stretched hairpin-like vortex element. Perhaps the earliest visually compelling evidence for this model came courtesy of Head \& Bandyopadhyay (1981), who performed flow-visualization experiments in a fog-filled boundary layer, illuminated by cross-stream laser sheets arranged at both $45^{\circ}$ and $135^{\circ}$ to the principle flow direction. At $45^{\circ}$, the recorded images exhibit distinctive elongated hoop-like features, whilst at $135^{\circ}$, the view provides a slice through what appear to be counter-rotating vortex pairs. These findings were entirely consistent with the model of hairpin vortical structures inclined at angles of approximately $45^{\circ}$ to the $x$-axis. It is with the work of Head \& Bandyopadhyay (1981) in mind that this present work was undertaken. The experiments described herein are largely similar, with the notable exception that rather than rely on qualitative information from flow-visualizations, the use of a stereoscopic particle image velocimetry (PIV) system has allowed us to obtain quantitative measurements of all three velocity components in the two inclined planes. Moin \& Kim (1985) and Adrian \& Moin (1988) have analysed $45^{\circ}$ inclined planes in LES and DNS domains. In both cases, distinct hairpin shapes were observed in the inclined vorticity vector fields.

Head \& Bandyopadhyay (1981) also performed visualizations in the streamwise/ wall-normal plane. The resultant view seemed to indicate that individual hairpin structures could occasionally group together in a remarkably ordered way to produce larger-scale flow features. They noted ramp-like agglomerations of hairpin structures stacking together at a characteristic growth angle of $15^{\circ}-20^{\circ}$. Such packet-like arrangements of hairpin structures have received considerable attention over the last five or so years. Zhou et al. (1999) used the simulated evolution of a hairpin-type 
structure (in low-Reynolds-number turbulent channel flow) to further investigate this phenomenon. As time is marched forward in the simulations, it is found that if the primary hairpin vortex is of sufficient circulation, it can spawn (or autogenerate) trailing and leading (secondary and tertiary) hairpin structures in the form of a coherent packet (i.e. a cohesive group of vortical structures in which all constituent entities convect downstream at the same approximate speed). Adrian, Meinhart \& Tomkins (2000b) further extended this packet view, with a series of streamwise/wallnormal plane PIV measurements. They found numerous instances where spanwise vortical structures (assumed to be the lifted heads of hairpins) were observed to convect downstream as a coherent group or packet and in a similar ramp-like arrangement to that observed by Head \& Bandyopadhyay (1981). Christensen \& Adrian (2001) anchored the observations of Adrian et al. (2000b) within a more statistical framework. Using linear stochastic estimation, they conditionally sampled the average flow field in the presence of spanwise swirling motions, revealing an inclined shear layer along which a series (packet) of negative signed vortices are arranged at an approximate inclination angle of $12^{\circ}-13^{\circ}$. Tomkins \& Adrian (2003) obtained PIV images in the streamwise/spanwise plane, revealing elongated lowspeed regions flanked by vortices (often in counter-rotating pairs). This picture is entirely consistent with the hairpin packet scenario. They also propose a scale-growth mechanism by which two packets can merge via vortex reconnection to form a larger event. Stereo PIV measurements in the same streamwise/spanwise plane were obtained by Ganapathisubramani, Longmire \& Marusic (2003), revealing a similar arrangement of elongated low-momentum regions and flanking vortical motions (believed to be the necks of hairpin structures). Using feature extraction algorithms, they were able to show that these elongated packet signatures are dominant contributors to the overall Reynolds shear stress.

In addition to experimental evidence, support for the hairpin model has also come from the theoretical framework of the attached eddy hypothesis. Perry \& Chong (1982) showed numerically that the induced velocity field due to a hierarchically scaled collection of simple representative hairpin-type vortices could successfully reproduce the salient features of the turbulent boundary layer. Perry \& Marusic (1995) and Marusic \& Perry (1995) performed quantitative calculations using the attached eddy hypothesis to show very good agreement with second-order statistics including spectra. A primary conclusion from this work was that general wall-bounded flows have a two-part wall wake-structure, with the wake component eddies becoming the dominant contributors in strong adverse pressure gradient flows. Marusic (2001) further refined the attached eddy hypothesis, showing that packet-type arrangements of the input structures could describe better the longer tails found in experimental two-point correlations.

Recognizing the hairpin vortex as an essential building block to turbulence, Acarlar \& Smith $(1987 a, b)$ set out to study its behaviour and evolution in a laminar boundary layer (so-called 'kernel' experiments). Hairpin vortices are artificially introduced into initially laminar boundary layers. It is shown that these wholly synthetic flows can successfully reproduce many of the observed and measured phenomena of fully developed turbulent boundary layers (including, but not limited to, low-speed streaks, ejections, sweeps, oscillations, break-up, regeneration of secondary structures and a general modification of mean-flow profiles towards those associated with turbulence). At the time, these experiments (along with Perry \& Chong's (1994) calculations) proved remarkably persuasive in cementing the view of turbulent boundary layers as a collection of hairpin vortical structures. 

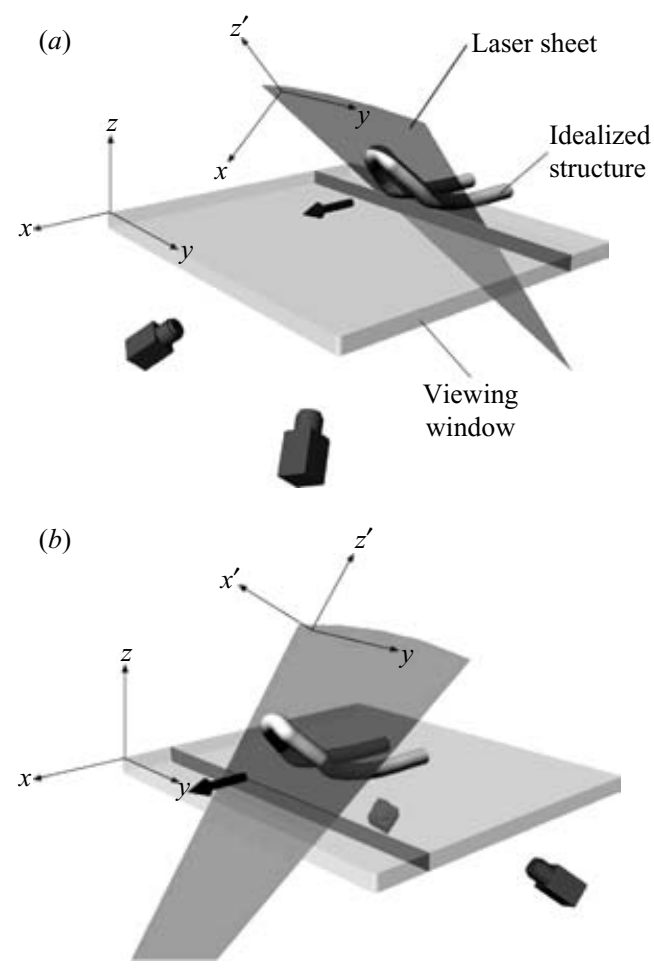

FIGURE 1. Basic PIV set-up for $(a) 45^{\circ}$ and $(b) 135^{\circ}$ cases, showing viewing window, laser sheet, camera location and representative idealized structure.

The objective of the current study is to exploit the possibilities of stereo PIV (in particular the ability of this technique to capture out-of-plane velocity vectors) to reveal the spanwise/wall-normal form of the coherent structure in fully developed turbulent boundary layers. In particular, we seek to address long-standing questions of structural symmetry and scaling, and use this unique plane to add to the views recently obtained from spanwise/streamwise PIV measurements (Ganapathisubramani et al. 2003; Tomkins \& Adrian 2003), and from streamwise/wall-normal planes (Adrian et al. 2000b; Christensen \& Adrian 2001).

\section{Facility}

Experiments are conducted in an open return suction-type boundary-layer windtunnel of working section $4.7 \times 1.2 \times 0.3 \mathrm{~m}^{3}$. Full details of the tunnel and the PIV system can be found in Ganapathisubramani et al. (2003). The flow was seeded with olive oil droplets from an array of eight Laskin nozzles located upstream of the tunnel inlet. The seeded flow is illuminated by pulsed sheets from two Nd:YAG lasers (Spectra Physics Quanta-Ray tuned to give $120 \mathrm{~mJ}$ per pulse) and imaged using a pair of TSI Powerview $2048 \times 2048$ pixel resolution digital cameras. The images are processed using unmodified commercial software (TSI Insight v6.1). Other than the orientation of the laser sheet/image plane, and the sheet thickness (which in this case was increased to approximately $1.5 \mathrm{~mm}$ ) the experimental facility used is identical to that described in Ganapathisubramani et al. (2003). The basic configuration for the $45^{\circ}$ and $135^{\circ}$ cases is shown in figure 1 . These figures clarify the transformed 


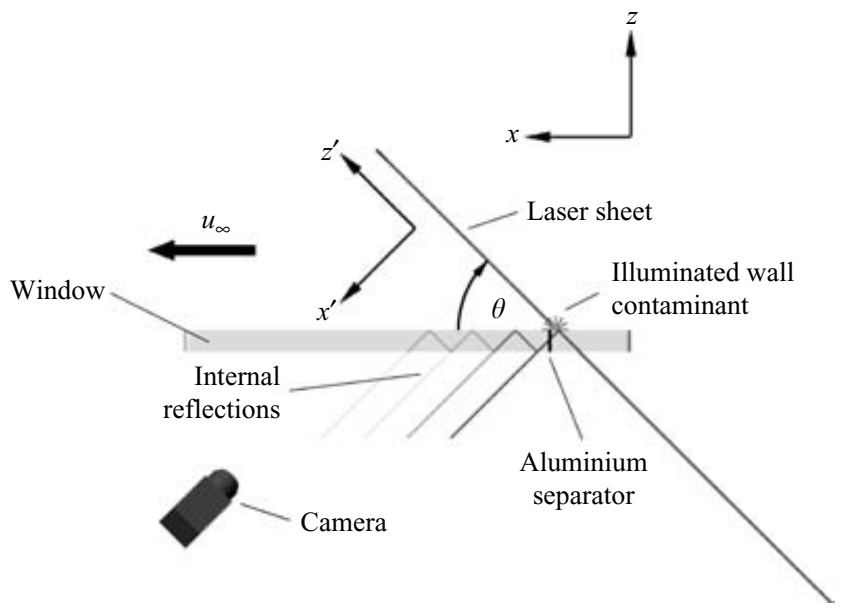

FIGURE 2. Side-view schematic of experimental set-up for the $45^{\circ}$ case, with representation of wall contamination and internal reflections.

axis system that will be used throughout the discussion. For both the $45^{\circ}$ and $135^{\circ}$ inclined planes, the out-of-plane direction is referred to as $x^{\prime}$, spanwise ordinates are unchanged $\left(y^{\prime}=y\right)$ and the third ordinate $z^{\prime}$ is defined appropriately by the righthand rule. The velocity components along the $x^{\prime}, y^{\prime}$ and $z^{\prime}$ axes are denoted as $u_{\theta}, v_{\theta}$ and $w_{\theta}$, respectively, where $\theta$ is the image-plane inclination angle $\left(45^{\circ}\right.$ or $\left.135^{\circ}\right)$. It is these components that are measured during the experiment. Streamwise, spanwise and wall-normal velocity components are recovered from simple trigonometric conversion.

$$
\begin{array}{ccc}
u=\frac{u_{45}+w_{45}}{\sqrt{2}}, & v=v_{45}, & w=\frac{w_{45}-u_{45}}{\sqrt{2}}, \\
u=\frac{u_{135}-w_{135}}{\sqrt{2}}, & v=v_{135}, & w=\frac{w_{135}+u_{135}}{\sqrt{2}} .
\end{array}
$$

Optical access for the laser sheet and cameras is provided by a glass viewing window set into the tunnel floor. Introducing the laser at an oblique $45^{\circ}$ angle to the glass, establishes a series of internal reflections that will appear as an array of bright fringes in the imaged PIV frames. Near the wall, these fringes can saturate the CCD and prevent the accurate capture of velocity vectors. In addition, however carefully the internal surface of the glass (forming the wall) is cleaned, the introduction of a seeded flow will eventually lead to contamination of this surface. At the location where the sheet is introduced, any contamination adhering to the surface will be illuminated, appearing as bright spots in the digitized images, and thus can also wash out the CCD in the near-wall region. These issues are represented schematically in figure 2. To address these problems, the window is formed from an assembly of two separate pieces of glass, separated by a thin sheet of aluminium shim. The aluminium shim serves a dual purpose. Internal reflections from the laser-access portion of the window are prevented from passing into the camera viewing section. In addition, the shim directly shields the cameras from any illuminated contaminant that has settled on the inner surface of the glass. By such methods, it is possible to obtain accurate measurements fairly close to the wall (conservative estimate of $z^{+} \approx 20-30$ ) for the two lowest-Reynolds-number cases. 


\begin{tabular}{|c|c|c|c|c|c|c|c|c|c|}
\hline \multirow[b]{2}{*}{$R e_{\tau}$} & \multirow[b]{2}{*}{$R e_{\theta}$} & \multirow{2}{*}{$\begin{array}{c}\delta \\
(\mathrm{m})\end{array}$} & \multirow{2}{*}{$\begin{array}{c}U_{\infty} \\
\left(\mathrm{m} \mathrm{s}^{-1}\right)\end{array}$} & \multirow{2}{*}{$\begin{array}{c}U_{\tau} \\
\left(\mathrm{m} \mathrm{s}^{-1}\right)\end{array}$} & \multicolumn{2}{|c|}{$\begin{array}{l}\text { Number } \\
\text { of frames }\end{array}$} & \multicolumn{2}{|c|}{$\begin{array}{l}\mathrm{d} T \\
(\mu \mathrm{s})\end{array}$} & \multirow{2}{*}{$\begin{array}{l}\text { Interrogation } \\
\text { spot size } \\
\text { (wall units } i^{+} \text {) }\end{array}$} \\
\hline & & & & & $45^{\circ}$ & $135^{\circ}$ & $45^{\circ}$ & $135^{\circ}$ & \\
\hline 690 & 1430 & 0.0764 & 3.09 & 0.138 & 1040 & (1040) & 207 & (227) & 15 \\
\hline 1010 & 2680 & 0.0792 & 5.29 & 0.210 & 1040 & (1040) & 127 & $(97)$ & $24 \dagger$ \\
\hline 1840 & 4740 & 0.0738 & 10.12 & 0.376 & - & (1040) & - & (67) & 43 \\
\hline 2800 & 7440 & 0.0692 & 17.26 & 0.608 & 1040 & (1040) & 37 & (37) & 69 \\
\hline
\end{tabular}

For each inclined plane configuration, experiments were repeated across a range of Reynolds numbers. As the free-stream velocity of the tunnel is increased for the higher $R e$ cases, it is necessary to reduce the time separation between image pairs $(\mathrm{d} T)$. A simple calculation based on the laser-sheet thickness and the mean out-ofplane velocity component served as a guide in this adjustment. An often quoted guide is that the out-of-plane particle displacement between image pairs should be less than one quarter of the light-sheet thickness (e.g. Adrian 1991). In reality, we are faced with the compromise of satisfying this condition, whilst maintaining a reasonable mean pixel displacement and large enough field of view. A Monte Carlo simulation was used to optimize these conditions and validate experimental accuracy.

The experimental conditions, along with key PIV parameters and statistics, are presented in table 1 . The PIV field of view extended only to approximately $80 \%$ of the boundary-layer thickness $(\delta)$. Hence, the $\delta$ values presented in table 1 were determined from a Coles law of the wall/wake fit to mean velocity profiles obtained using a Pitot-static tube. The friction velocity $\left(U_{\tau}\right)$ is obtained from a Clauser chart fit (with $\log$ law constants $\kappa=0.41$ and $A=5.0$ ). The magnification and the interrogation window size $(i)$ were held constant in physical units for all four Reynolds numbers tested $\left(32 \times 32\right.$ pixels $\left.\sim 1.6 \times 1.6 \mathrm{~mm}^{2}\right)$. Thus, in terms of viscous wall units, the inner scaled interrogation window $i^{+}\left(=i U_{\tau} / \nu\right.$, where $v$ is the kinematic viscosity) increases in size from 15 to 69 wall units as $R e_{\tau}\left(=\delta U_{\tau} / v\right)$ increases from 690 to 2800 . In Kolmogorov scales $(\eta)$, this represents an interrogation window in the log region (at $z^{+}=100$ ) that increases with Reynolds number from approximately $6 \eta$ to $25 \eta$ (as $R e_{\tau}$ increases from 690 to 2800). An overlap of $50 \%$ was used to calculate vectors, yielding an inner scaled vector resolution of half the quoted interrogation spot size.

\section{Validation mean profiles}

For clarity of presentation, the mean-flow data in this section are down-sampled to 30 wall-normal locations (logarithmically spaced for figure 3 , and equally spaced for figures 4 and 5). Throughout the paper capitalized velocities (e.g. $U$ ) and overbars indicate time-averaged values, lower case $(u)$ denotes fluctuating components and the prime symbol for velocities $\left(u^{\prime}\right)$ denotes r.m.s. values. Figures $3(a)$ and $3(b)$ give the mean velocity profiles for the $45^{\circ}$ and $135^{\circ}$ cases for all $R e_{\tau}$ tested. The profiles compare well and exhibit the expected Reynolds-number behaviour in both the log and wake regions. Figure 3(c) offers a direct comparison between velocity profiles for both inclined planes at $R e_{\tau}=1010$ (which is the data-set on which the majority of 
(a)

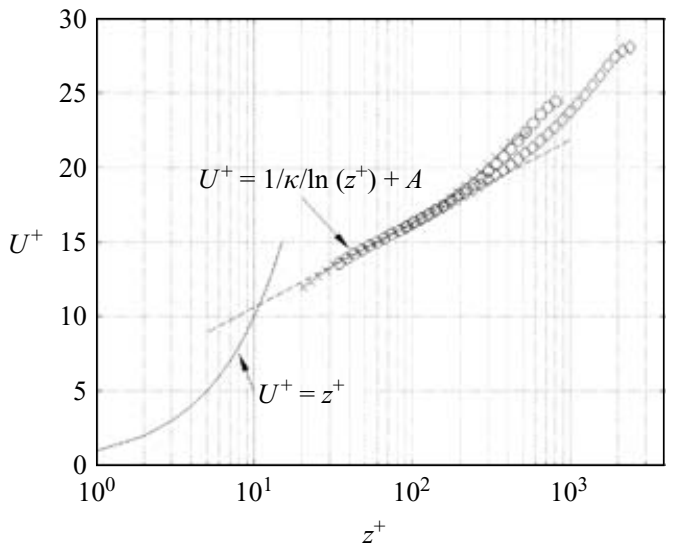

(b)

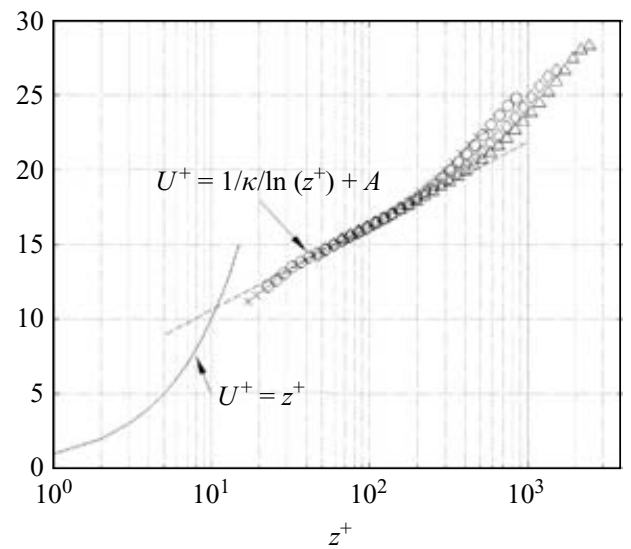

(c)

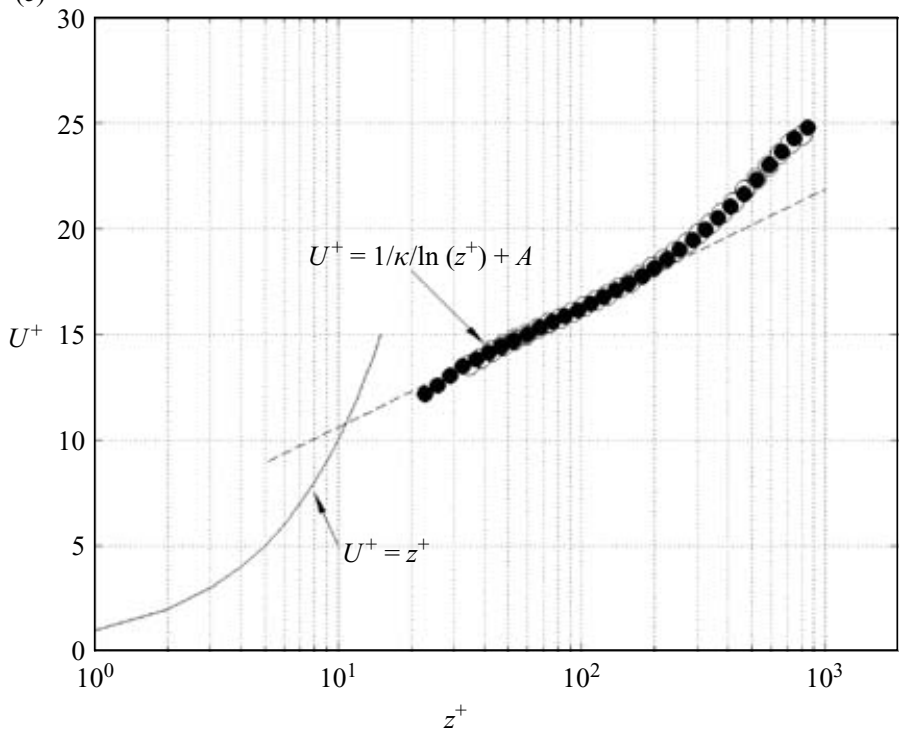

FiguRE 3. Mean velocity profiles for $(a) 45^{\circ}$ case $(b) 135^{\circ}$ case. $\times, R e_{\tau}=690 ; \bigcirc, R e_{\tau}=1010$; $\diamond, R e_{\tau}=1840 ; \triangle, R e_{\tau}=2800$. Plot (c) shows a comparison between $45^{\circ}$ (open) and $135^{\circ}$ (closed) planes at $R e_{\tau}=1010$. For clarity of presentation, experimental mean-flow data are down-sampled to $30 \mathrm{log}$-spaced wall-normal locations.

the proceeding analysis will be based). The good agreement between the two profiles is clearly evident.

Figure 4 gives r.m.s. turbulent intensity profiles for the three velocity components. The plots on the left-hand side of the page show the $45^{\circ}$ case whilst the right-hand side shows the $135^{\circ}$ plane. By way of comparison, these are plotted against intensities found from direct numerical simulations performed by Spalart (1988) at $\operatorname{Re}_{\theta}=1410$. This data-set is collated within Balint, Wallace \& Vukoslavcevic (1991). In general, the agreement between inclined plane PIV and existing DNS data is reasonable, given that we expect intensities to collapse with outer variables in the wake region (for all $\left.R e_{\tau}\right)$. However, it is noted that the inclined plane PIV has tended to under-resolve the turbulent intensities at all $R e_{\tau}$. Figure 5 shows the Reynolds shear stress profiles for the two inclined planes (again plotted against DNS data). For this statistic, 

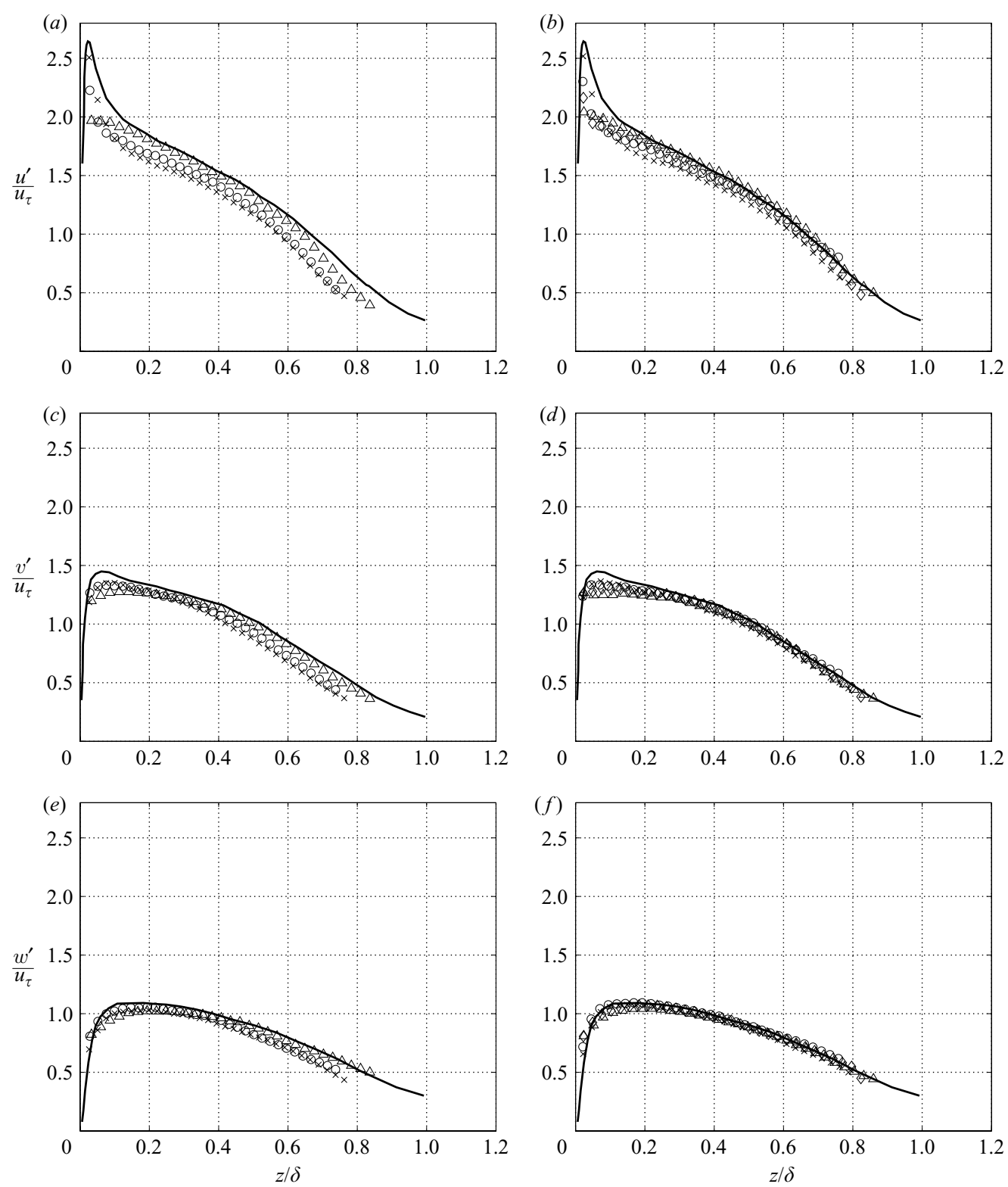

FIGURE 4. Root mean square turbulent intensity profiles for $(a) 45^{\circ} u^{\prime}$ (b) $135^{\circ} u^{\prime}$ (c) $45^{\circ} v^{\prime}$ (d) $135^{\circ} v^{\prime}(e) 45^{\circ} w^{\prime}(f) 135^{\circ} w^{\prime}$ case. $\times, R e_{\tau}=690 ; O, R e_{\tau}=1010 ; \diamond, \operatorname{Re}_{\tau}=1840 ; \triangle$, $R e_{\tau}=2800$. The solid line shows data from Spalart (1988). For clarity of presentation, experimental mean-flow data are down-sampled to 30 linearly spaced wall-normal locations.

there is a greater discrepancy between the PIV profiles and Spalart's results (any disparity in $u^{\prime}$ and $w^{\prime}$ will probably be exacerbated for $\overline{u w}$ ). The reasons why the inclined plane PIV data has tended to under-resolve the turbulent fluctuations are investigated more thoroughly in the error treatment of $\S 3.1$. However, the quality of agreement in figures 3 and 4 and the correct qualitative trend in figure 5 are taken as proof that, despite the large out-of-plane components, the current technique of inclined cross-plane stereoscopic PIV has successfully resolved the principle features 

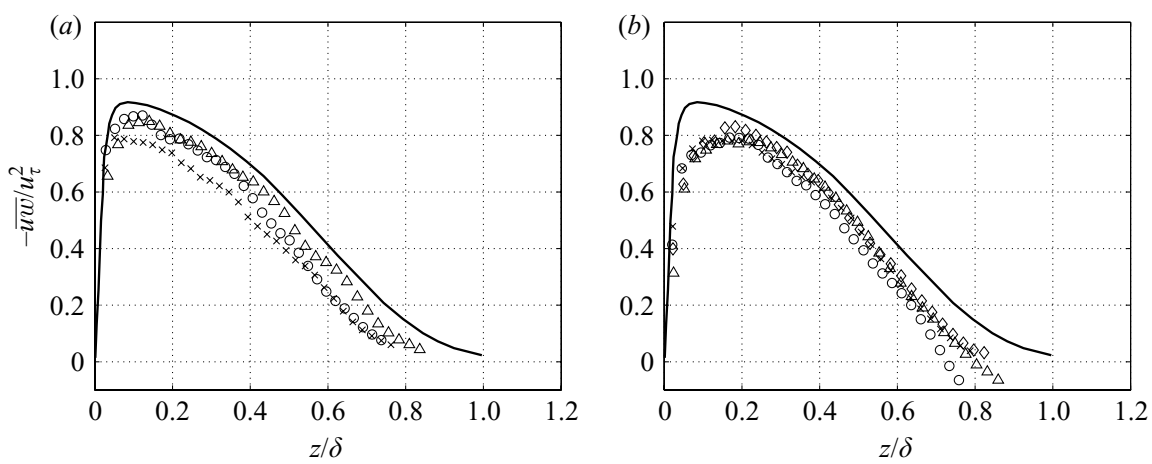

FIGURE 5. Mean Reynolds shear stress profiles for $(a) 45^{\circ}$ (b) $135^{\circ}$. Symbols and lines are as defined in previous figure.

of the turbulent boundary layer. As further validation Ganapathisubramani et al. (2005) have compared two-point correlations from the inclined-plane data-sets with existing PIV data taken in spanwise wall-normal planes (originally presented in Ganapathisubramani et al. 2003), showing excellent agreement between the two cases.

\subsection{Interrogation spot size}

To minimize the likelihood of missing vectors, the decision was made to fix the physical dimension of the interrogation window $(i)$ at $32 \times 32$ pixels for all experiments. This effectively acts as a spatial filter during cross-correlation, with any effect becoming increasingly pronounced for higher $R e$, as the inner-scaled interrogation window $i^{+}$ increases in size (see table 1). There is some evidence of such spatial resolution issues close to the wall, where Figures $4(a)-4(d)$ indicate that the inclined plane PIV has failed to fully resolve the near-wall peak in the $u$ and $v$ fluctuations. Such behaviour is expected, since at these wall normal stations the filter width due to $i^{+}$is comparable in size to the small-scale structures that reside (primarily) in the near-wall region. In keeping with this notion, a closer analysis of Figures $4(a)-4(d)$ reveals that the under-resolved energy in the near-wall $u$ and $v$ fluctuations is more pronounced for the higher-Reynolds-number cases. Regardless, for this study, the issue of spatial resolution is considered of secondary importance, since at no point are conclusions drawn from measurements made in the buffer region. Instead, we concentrate almost exclusively on the $\log$ and wake regions where we expect the filtering due to $i^{+}$to be minimal. As validation of this assumption, the $R e_{\tau}=1010$ images were reprocessed using a $64 \times 64$ interrogation window. An analysis of these results in $\S 6$ reveals that this doubling of the interrogation spot size has only marginal effects on the spanwise and wall-normal length scale results (see figures 17, 18 and 19). This is the only instance in the present analysis when the higher-Reynolds-number results (with the larger $i^{+}$) are used. All preceding analysis involving two-dimensional swirl calculations will concentrate exclusively on the $R e_{\tau}=1010$ data, where the boundary layer is fully developed and the spot size is considered sufficient to resolve swirling motions in the $\log$ and wake regions.

The wider underestimation of fluctuating statistics evident beyond the near-wall region in figures 4 and 5 is not believed to result from spatial resolution issues. It is argued in $\$ 3.2$ that this more general discrepancy results from a combination of conflicting errors due to limited pixel displacements, a limited dynamic range, peak locking effects and a residual pixel displacement error intrinsic to stereo PIV measurements. 


\subsection{Residual pixel displacement and peak locking error}

Large out-of-plane velocity components (as encountered during inclined plane measurements) place additional strain on the experimental accuracy of stereo PIV systems. There is a residual error unique to stereo PIV calculations, in which a least-squares method is used to solve four simultaneous pixel displacement equations to arrive at the three velocity components (in this case $u_{\theta}, v_{\theta}$ and $w_{\theta}$ ). This mean residual error ranges from 0.2 pixels near the wall to 0.15 pixels towards the edge of the boundary layer. This is higher than found for typical flow-orthogonal planes (where the out-of-plane velocity components are smaller). For example, Ganapathisubramani et al. (2005) found a residual error of just 0.05 pixels for streamwise/spanwise plane stereo PIV measurements. As $R e_{\tau}$ is changed, $\mathrm{d} T$ is altered such that the mean pixel displacements remain approximately constant, ranging from 5 pixels near the wall to 12 at the top of the image (approximately $0.8 \delta$ ). Hence this residual error causes uncertainties in the velocity measurements ranging from approximately $0.04 U$ near the wall, to $0.012 U$ towards the free stream. For the current experiments, it was deemed desirable to hold the spanwise dimension of the measurement frame at approximately $1.2 \delta$. With a fixed laser-sheet thickness of $1.5 \mathrm{~mm}$, this essentially limits the mean pixel displacements we can obtain (since particles should only be displaced by $0.25-0.30$ of the light-sheet thickness between image pairs). As a result, the mean displacements and especially the dynamic range are lower than normally considered ideal. As well as increasing the percentage velocity errors due to the previously mentioned residual error, smaller overall pixel displacements can exacerbate any error due to pixel locking. Even close to the wall, the streamwise r.m.s. fluctuation $\left(u^{\prime}\right)$ is less than 0.5 pixels for these experiments. Christensen (2004) has shown that the errors due to pixel locking become more acute for low mean pixel displacements, and that where fluctuating displacements have magnitudes of less than 0.5 pixels (which is the case here) this manifests particularly as an underestimation of the r.m.s. and Reynolds shear stress statistics. Despite using a Gaussian sub-pixel estimator, this appears to be the case with the present results, although the degree of error would imply that the data set is only marginally afflicted by peak-locking (somewhat similar to Christensen's Case IA). Certainly, the data in no way exhibit the degree of complete pixel locking that can cause large errors in the two-point correlation results (Christensen's Case 0A).

\section{In-plane swirl}

Using the two-dimensional form of the velocity gradient tensor, it is possible to calculate swirl (identified as the imaginary part of the complex eigenvalue). This process is described in Adrian, Christensen \& Lui (2000a) and provides a useful metric to highlight vortex cores. The only change here is that we calculate two-dimensional swirl from in-plane velocity components measured on the inclined planes.

$$
\left|\begin{array}{cc}
\frac{\partial v_{\theta}}{\partial y^{\prime}}-\lambda & \frac{\partial v_{\theta}}{\partial z^{\prime}} \\
\frac{\partial w_{\theta}}{\partial y^{\prime}} & \frac{\partial w_{\theta}}{\partial z^{\prime}}-\lambda
\end{array}\right|=0
$$

From which the following quadratic solution is obtained,

$$
\lambda=\frac{1}{2}\left(\frac{\partial v_{\theta}}{\partial y^{\prime}}+\frac{\partial w_{\theta}}{\partial z^{\prime}}\right) \pm \frac{1}{2} \sqrt{\underbrace{\left(\frac{\partial v_{\theta}}{\partial y^{\prime}}+\frac{\partial w_{\theta}}{\partial z^{\prime}}\right)^{2}}_{b^{2}}-\underbrace{4\left(\frac{\partial v_{\theta}}{\partial y^{\prime}} \frac{\partial w_{\theta}}{\partial z^{\prime}}-\frac{\partial w_{\theta}}{\partial y^{\prime}} \frac{\partial v_{\theta}}{\partial z^{\prime}}\right)}_{4 a c}} .
$$


Swirl $\left(\lambda_{c i}\right)$ is defined as the imaginary part of the quadratic solution, in regions where the solution is complex. In other words,

$$
\lambda_{c i}=\frac{1}{2} \sqrt{\left|b^{2}-4 a c\right|} \text { where } 4 a c>b^{2}
$$

In this form, swirl has no sign information. Sign is recovered by multiplying $\lambda_{c i}$ with the sign of the local in-plane vorticity,

$$
\lambda_{s}=\lambda_{c i}\left(\frac{\omega_{x^{\prime}}}{\left|\omega_{x^{\prime}}\right|}\right) .
$$

For the higher-Reynolds-number experiments, there is a danger that the larger interrogation spot-size will not fully resolve all vortex cores. Hence, discussion here is limited to swirl results from the $R e_{\tau}=1010$ experiment. Figure 6 shows example instantaneous flow fields for both inclined planes at this Reynolds number. It must be noted that the $45^{\circ}$ and $135^{\circ}$ measurements are from separate experiments (made at different times). The two example frames are chosen on the basis that each contains similar evidence of large plumes of low $u$ momentum erupting from the wall. These negative $u$ fluctuations are the lighter shaded regions on figures $6(a)$ and $6(b)$ and are typical of many instantaneous observations. The streamwise velocity fluctuations of figures $6(a)$ and $6(b)$ together with the in-plane vector field of figures $6(c)$ and $6(d)$ give a good indication of the typical complexity in the flow. However, with careful examination, some interesting recurrent features emerge. Such low-speed regions are actually strong ejection events $(-u,+w)$ and they are typically flanked by darker shaded regions signifying high-speed sweep events $(+u,-w)$. Together these sweep and ejection regions form a large positive contribution to the Reynolds shear stress (approximately 12 times larger than the mean peak shown in figure 5). Figures 6(e) and $6(f)$ show example in-plane swirl patterns for both inclined planes. Darker shaded patches show regions of positive signed swirl, whilst the white areas indicate negative. From this viewpoint (viewed along the negative $x^{\prime}$ direction), positive swirl implies a counterclockwise rotating vector field. A careful comparison with the inplane vector fields (figure $6 c, d$ ) reveals that two-dimensional swirl is successfully identifying rotating patterns in the flow. Such patterns are associated with vortex cores piercing the planes (at some oblique angle). It is immediately noticeable from a comparison of the left- and right-hand-sides of figure $6(c-f)$ that the in-plane swirl is much more prevalent in the $135^{\circ}$ plane than in the $45^{\circ}$. This is consistent with the observations of Head \& Bandyopadhyay (1981) who noted considerable in-plane vortical activity in their $135^{\circ}$ flow visualizations as opposed to the corresponding $45^{\circ}$ images, which exhibited mainly elongated hoop-like patterns. Such patterns are entirely consistent with the inclined structures proposed by the hairpin vortex and packet models. This disparity in swirling motions can be highlighted by calculating the mean swirl fluctuation at all wall-normal locations across the boundary layer for both planes. This result, along with the r.m.s. in-plane vorticity as a function of $z$, is shown in figure 7 . In this case, we can only calculate the vorticity based on in-plane velocity gradients $\left(\omega_{x^{\prime}}\right)$. However, the r.m.s. levels shown in figure $7(b)$ are consistent with those reported for streamwise and wall-normal vorticity fluctuations in Honkan \& Andreopoulos (1997). Averaging across the data set in this way clarifies our initial observation that there is substantially less in-plane swirling activity in the $45^{\circ}$ vector fields. The plots reveal that this disparity is greatest near the wall. 

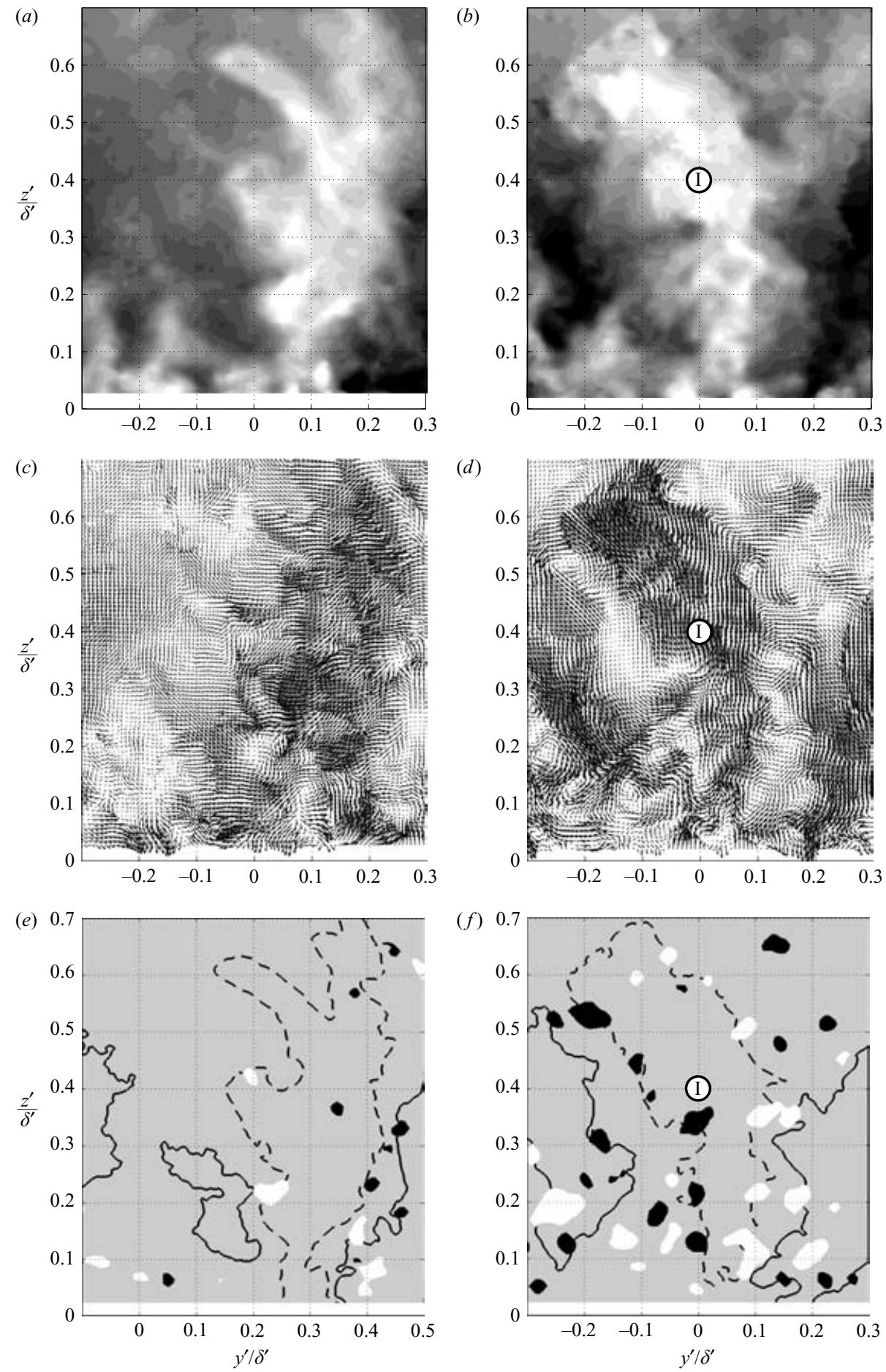

FIGURE 6. Example instantaneous flow fields for $45^{\circ}$ (left-hand side) and $135^{\circ}$ (right-hand side) experiments at $R e_{\tau}=1010$. (a) and (b) show streamwise fluctuating velocity $u$. Grey scale ranges from (white) $u / U_{\tau}=-4$ to (black) $u / U_{\tau}=4$. $(c)$ and $(d)$ show instantaneous in-plane vector fields produced from $v_{\theta}$ and $w_{\theta} .(e)$ and $(f)$ show instantaneous in-plane signed swirl. Shading shows positive and negative swirl, (dark) $\lambda_{s}^{+}>0.1$, (light) $\lambda_{s}^{+}<-0.1$. Lines indicate contours of streamwise velocity fluctuations; (dashed) $u<-1.5 U_{\tau}$; (solid) $u>1.5 U_{\tau}$. 

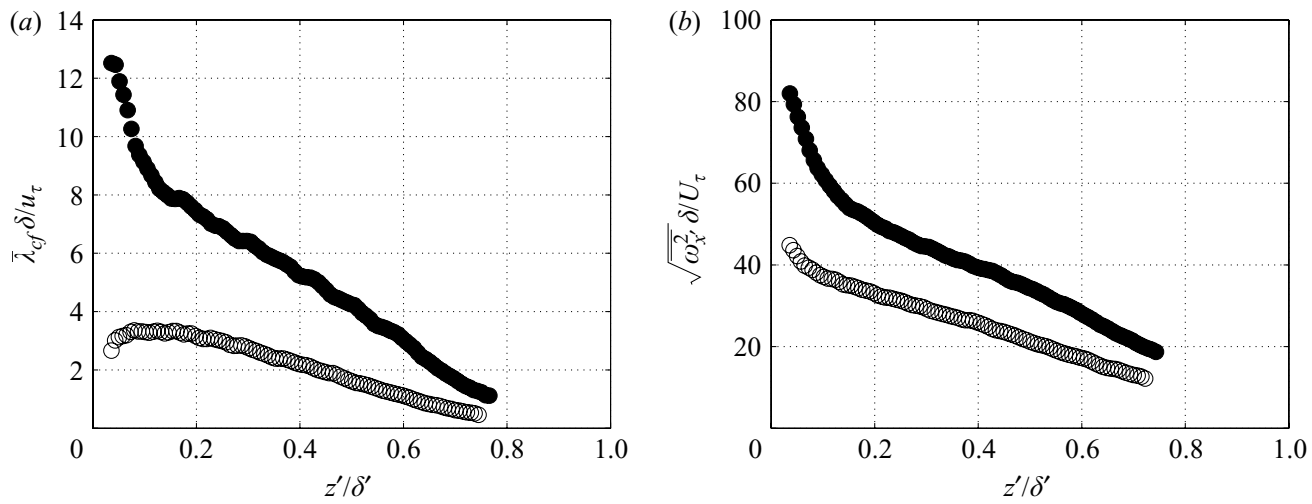

FIGURE 7. Wall-normal variation of inner-scaled $(a)$ Mean swirl and $(b)$ root mean square in-plane vorticity for (open) $45^{\circ}$ and (closed) $135^{\circ}$ cases at $R e_{\tau}=1010$.

(a)

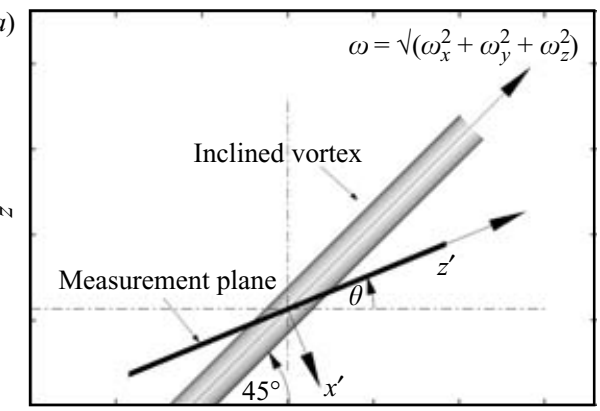

(c)

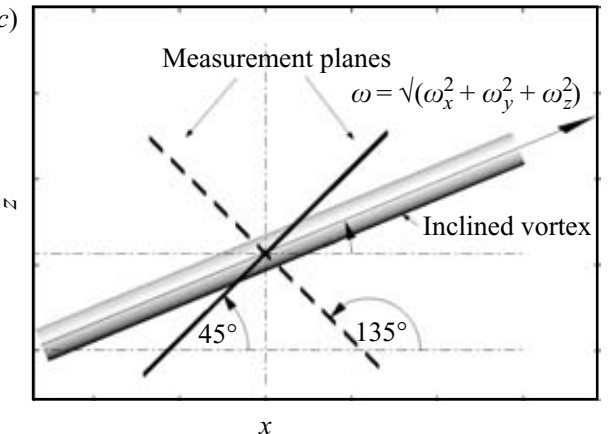

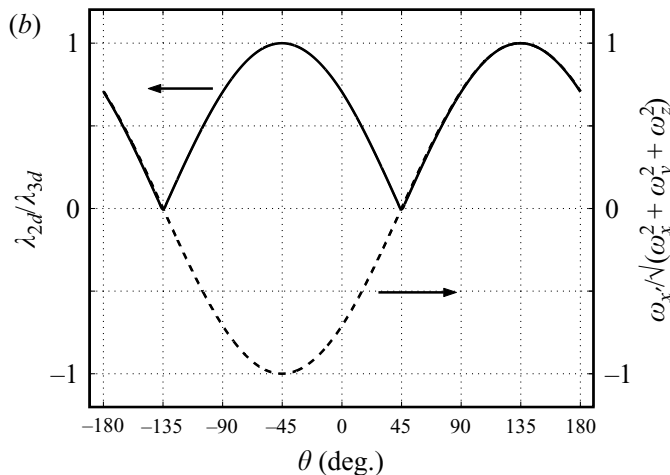

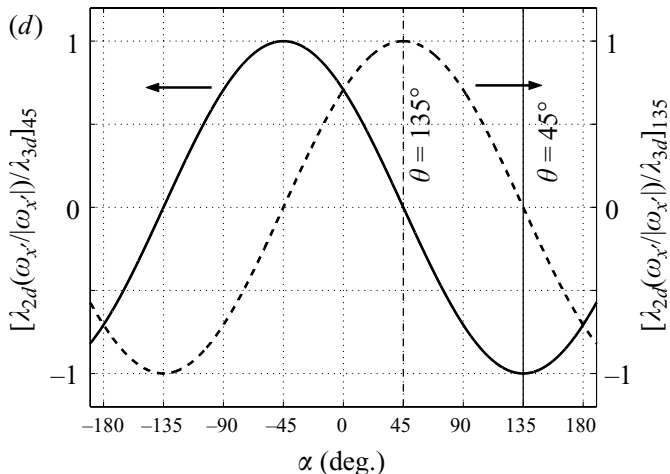

FIGURE 8. (a) $\alpha=45^{\circ}$ inclined vortex system bisected by the measurement plane at various angle $\theta ;(b)$ variation with $\theta$ of (solid) $\lambda_{2 d} / \lambda_{3 d}$ and (dashed) $\omega_{x^{\prime}} / \sqrt{ }\left(\omega_{x}^{2}+\omega_{y}^{2}+\omega_{z}^{2}\right) ;(c) \theta=45^{\circ}$ and $135^{\circ}$ measurement planes with vortex at various inclination angles $\alpha ;(d)$ variation of signed $\lambda_{2 d}$ with $\alpha$ as registered in the $\theta=45^{\circ}$ and $135^{\circ}$ measurement planes.

\subsection{Limits of the two-dimensional swirl approximation}

Using the Biot-Savart law, the induced velocities due to a simple $45^{\circ}$ inclined vortex are calculated onto planes that cut the vortex across the range of angles $0^{\circ}<\theta<180^{\circ}$. This scenario is shown in figure $8(a)$, with the plane drawn at $\theta \approx 20^{\circ}$. Using this model, we can directly compare the two-dimensional in-plane swirl approximation 
$\left(\lambda_{2 D}\right)$ with swirl calculated from the full three-dimensional gradient tensor $\left(\lambda_{3 D}\right)$. The solid line on figure $8(b)$ shows the ratio between the maximum calculated three-dimensional and two-dimensional swirl across the range of, measurement plane inclination angles $\theta$. Three-dimensional swirl is independent of the axis system used, and therefore invariant with $\theta$. Thus, figure $8(b)$ provides a measure of the accuracy of the two-dimensional approximation. It is clear that for $\theta=45^{\circ}$ (when the vortex axis lies in the plane), the two-dimensional swirl completely fails to register the event. Conversely, at $\theta=135^{\circ}$, the two-dimensional approximation exactly matches the full three-dimensional calculation. The dashed line of figure $8(b)$ shows the in-plane vorticity component $\omega_{x^{\prime}}$ at the core of the inclined structure (normalized by the threedimensional vorticity magnitude at the core). As for swirl, it is noted that the in-plane vorticity component exactly captures the three-dimensional vorticity at $\theta=135^{\circ}$ (and recovers none of the vorticity at $\theta=0$ ). More importantly, $\omega_{x^{\prime}}$ changes sign at $45^{\circ}$. Recall from (4.4), that the sign of this quantity is used to determine the sign for $\lambda_{s}$. Hence $\lambda_{s}$ becomes unreliable when the vortex inclination angle $(\alpha)$ is close to the measurement plane inclination angle $(\theta)$. The curves on figure $8(b)$ were calculated numerically. However, we can solve the two-dimensional and three-dimensional swirl equations analytically for the scenario shown (using the Biot-Savart law to give the velocity gradients). In this way, it is found that the swirl ratio of figure $8(b)$ is actually given by the functional form $|\cos (\theta+45)|$, whilst the in-plane vorticity ratio is given by $-\cos (\theta+45)$. Calculations were repeated for curved vortex tubes and found to have negligible effect on the result.

Figure $8(d)$, shows $\lambda_{2 D}$ due to vortices at various angles $\alpha$ as measured by the inclined planes at $45^{\circ}$ and $135^{\circ}$. This is representative of the actual experimental configuration. Each plane registers a maximum swirl for vortices perpendicular to the plane (at $\alpha=\theta \pm 90$ ). Note that both planes register the same in-plane swirl at $\alpha=0^{\circ}$ (same value and sign) and $\alpha=90^{\circ}$ (same value, opposite sign). In situations where $\alpha>45^{\circ}$, the same event is registered as opposite signed swirl in the $45^{\circ}$ plane. This figure highlights the inclined nature of the coherent structure. From figure $7(a)$, the near-wall mean swirl in the $135^{\circ}$ plane is approximately three times that of the $45^{\circ}$ plane. Figure $8(d)$ shows that such a scenario will arise at vortex inclinations $\alpha=26^{\circ}$ or $\alpha=63^{\circ}$.

In summary then, it must be kept in mind that the two-dimensional swirl approximation will show low values for either no swirling activity or for an eddy whose axis lies in the plane. In any case, the disparity between the mean occurrence of two-dimensional swirl in the $45^{\circ}$ and $135^{\circ}$ planes shown in figure 7 remains a valid indication of an inclined structural tendency.

\subsection{The hairpin/hairpin packet model}

A sketch of an idealized hairpin packet is shown in figure 9. This can be used to explain some of the phenomena noted in the example forward and backwards planes of figure 6, and the averaged swirl results of figure 7. The individual hairpin structures are depicted at an inclination angle that is close to $45^{\circ}$ (but varying with wall-normal distance). The two inclined planes bisecting this packet show that if such an arrangement of structures were indeed typical, we would expect to find greater evidence of in-plane swirling activity in the $135^{\circ}$ plane than the $45^{\circ}$ case (in fact, if all structures were ideal and aligned at $45^{\circ}$ to the streamwise axis, $\S 4.1$ has shown that there would be no two-dimensional swirling activity evident in the $45^{\circ}$ plane). Furthermore, the packet arrangement would seem to imply the occasional occurrence in the $135^{\circ}$ plane of well-ordered arrangements of positive and negative swirl, stacked 


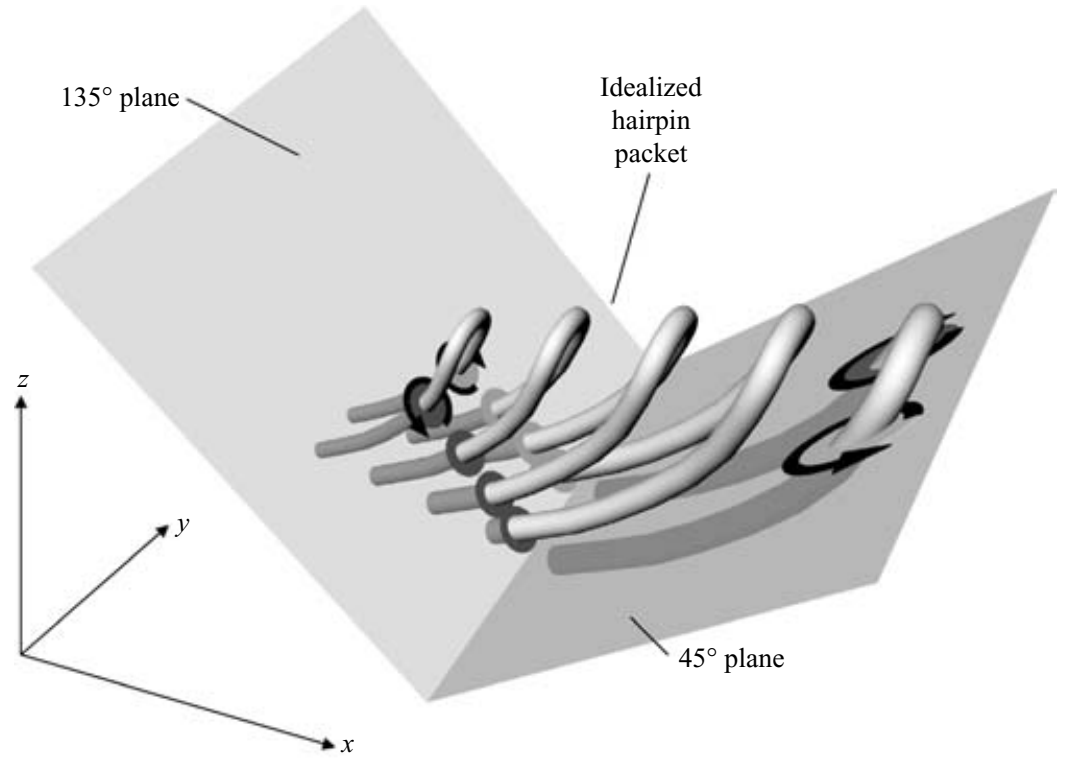

FIGURE 9. Schematic rendering of an idealized hairpin vortex packet. Both $45^{\circ}$ and $135^{\circ}$ image planes are represented to highlight occurrence of in-plane swirling motions. Shaded patches show positive and negative in-plane swirl; (dark) $\lambda_{s}>0$; (light) $\lambda_{s}<0$.

above one another, and flanking a region of reduced streamwise momentum. The negative $u$ region region labelled I in figure $6(b, d, f)$, is a clear example of such a packet signature. Certainly, this region is qualitatively similar to the flow pattern shown in the $135^{\circ}$ plane of figure 9 , with predominantly positive swirling motions to the left of region I and negative swirling motions to the right. Similar patterns of positive and negative swirling motions flanking elongated low-speed regions were noted previously in spanwise/streamwise plane PIV results (Ganapathisubramani et al. 2003; Tomkins \& Adrian 2003). The real advantage of the current inclined data set is that we are also afforded some insight into the wall-normal extent of such features. Region I in figure $6(b)$ extends beyond half the boundary-layer thickness. If we accept the classic packet form depicted in figure 9, in particular the ramp-like shape (see Head \& Bandyopadhyay 1981; Adrian et al. 2000b), the implication is that the uppermost structure in figure $6(f)$ region I is actually the most upstream structure piercing the plane. This will also be the smallest structure in the group. Hence region I may actually be the signature of a far larger packet (that extends further still into the turbulent boundary layer downstream of the measurement plane).

The individual hairpin structures that comprise the packet of figure 9 have an inclination angle which increases with height, becoming ever more upright towards the head. Although the figure is merely an idealized sketch, this shape has some basis in the literature (Head \& Bandyopadhyay 1981; Acarlar \& Smith 1987a,b; Zhou et al. 1999). It is clear from the light and dark swirl patches shown in figure 9 , that when the vortex inclination angle $\alpha>45^{\circ}$, the counter-rotating swirl signature due to the hairpin in the $45^{\circ}$ plane will turn in the opposite sense to that in the $135^{\circ}$ plane. This was previously shown analytically by the signed swirl plots of figure $8(d)$, with the two-dimensional swirl in the $45^{\circ}$ plane (solid line) changing sign when $\alpha$ exceeds $45^{\circ}$.

An alternative scenario to hairpin packets to explain the ejecting low-speed region (labelled I in figure $6(b, d, f)$ ) is the notion of the 'superburst' as proposed by $\mathrm{Na}$, 
Hanratty \& Liu (2001), who considered low-Reynolds-number DNS data. Liu, Adrian \& Hanratty (2001) conducted proper orthogonal decomposition (POD) on PIV data, finding that large-scale motions (captured by the first few eigenmodes) contained a large fraction of the total kinetic energy due to the streamwise velocity fluctuations. Further analysis of DNS data showed these large-scale motions to be 'plumes' of lowspeed fluid erupting from the viscous wall layer and extending a considerable distance towards the channel centreline. Wallace (1985) presents earlier photographic evidence of such large-scale 'plumes', originating in the sublayer and traversing the entire boundary layer (see p. 259), although in this instance these features were attributed to the growth of vortical structures. Our own instantaneous measurements on inclined $135^{\circ}$ planes indicate that swirl events commonly flank such 'plumes'. This suggests that the hairpin packet model may provide a basis for such events, with ejecting low-speed regions occurring within the packet (between the legs of nested hairpin vortices). It is probable that with sufficiently high $R e_{\tau}$ the 'superbursts' occurring in the DNS data-sets would be seen to be flanked by swirl events (certainly spanwise vortices are clearly visible crowning the streamwise/wall-normal plane 'superburst' examples shown in $\mathrm{Na}$ et al. 2001). The principal difference between the superburst and hairpin packet models lies in the significance placed on given flow features. The notion of superbursts tends to focus attention on the elongated regions of lowmomentum (ejecting) fluid, whilst the packet scenario places greater emphasis on the vortical structure. There is a subtle cause and effect issue inherent in these two views. The question of whether hairpin vortices cause the regions identified by Hanratty and co-workers as 'superbursts', or whether hairpins form over pre-existing lifted low-momentum regions or 'plumes', cannot be answered definitively by the current data-set and almost certainly requires a time-resolved view.

\section{Conditional analysis}

Thus far the discussion has been based on evidence contained in instantaneous velocity fields. In the proceeding analysis, attempts will be made to reinforce these observations using statistical techniques.

\subsection{Detection on swirl}

First, we consider the ensemble-averaged swirl field, sampled conditionally on the occurrence of swirl of a given sign,

$$
\left\langle\begin{array}{cc}
\lambda_{s}\left(y^{\prime}, z^{\prime}\right) \mid \begin{array}{c}
\lambda_{s}\left(0, z_{\text {ref }}^{\prime}\right)>t h \\
\lambda_{s}\left(0, z_{r e f}^{\prime}\right)<-t h
\end{array}
\end{array}\right\rangle .
$$

Angled brackets indicate an ensemble average based on some given detection criteria. This condition is specified at a certain wall-normal location $\left(z_{r e f}^{\prime}\right)$. A threshold $(t h)$ is defined to prevent erroneous detection on weak swirling motions (often associated with noise in the vector field). Figure 10 shows the ensemble-averaged swirling field resulting from such a conditional analysis (with $\left.\lambda_{s}\left(0, z_{r e f}^{\prime}\right)>t h\right)$. Results are shown for the $135^{\circ}$ inclined plane at various values of $z_{\text {ref }}$. Given the input condition, there has to be a positive signed swirl event located at $\left(0, z_{\text {ref }}\right)$ in the ensemble-averaged field. However, beyond this point, any wider coherence has not been preordained by the input condition, and must be representative of some wider flow feature. If in-plane vortex cores have a random distribution in the vector fields (with no preferred grouping tendency) there would be a uniform probability of positive or negative signed swirl occurring about our detection condition. This would show as 

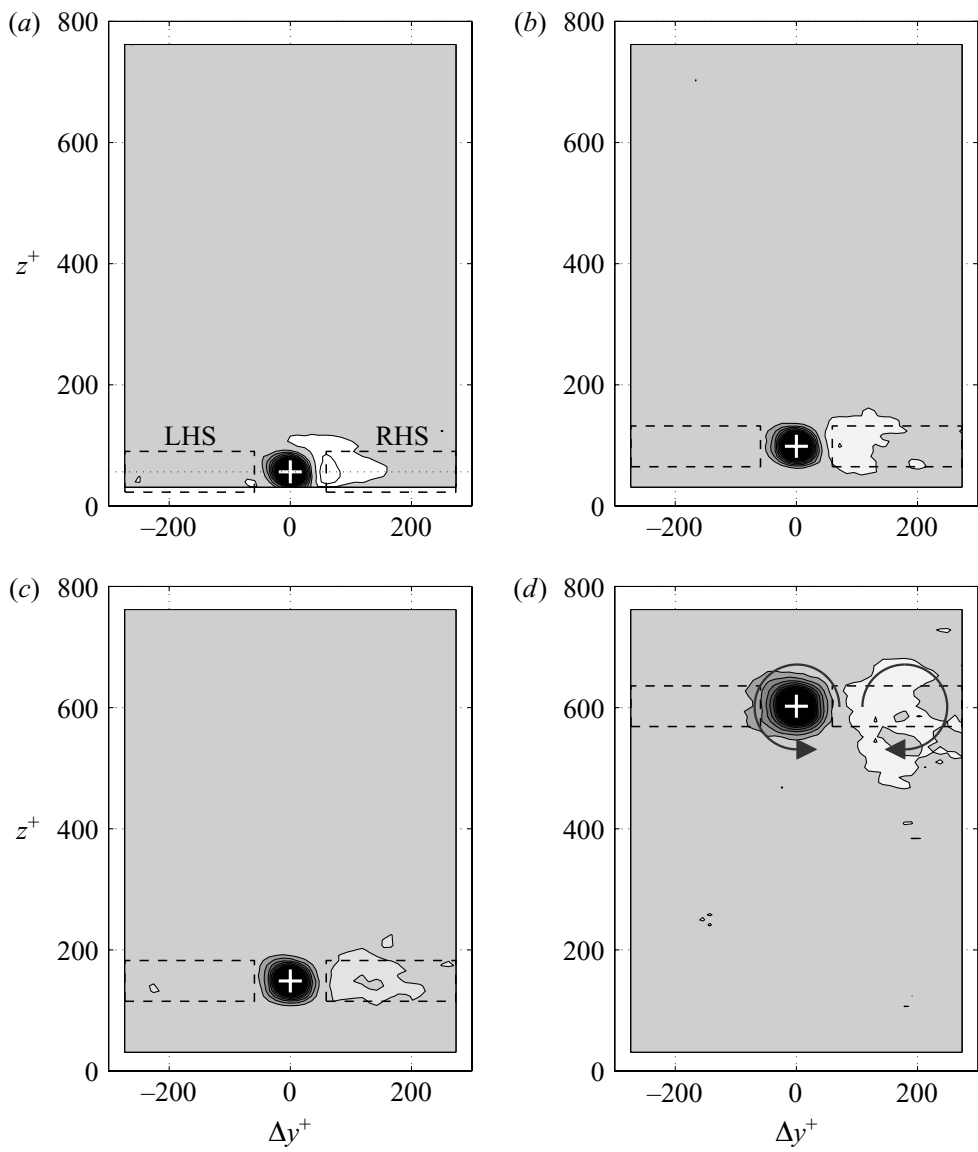

FIGURE 10. Ensemble-averaged signed swirl, conditionally sampled upon occurrence of positive swirl at $z_{\text {ref }}\left(\left\langle\lambda_{s}\right\rangle\right.$ given $\left.\lambda_{s}\left(0, z_{\text {ref }}^{\prime}\right)>t h\right)$. Results shown are for the $135^{\circ} R e_{\tau}=1010$ case; (a) $z_{\text {ref }}^{+}=50 ;(b) z_{\text {ref }}^{+}=100 ;(c) z_{\text {ref }}^{+}=150 ;(d) z_{\text {ref }}^{+}=600$. Contour levels from $\left\langle\lambda_{s}\right\rangle / \overline{\lambda_{c i}}=-0.4$ to 0.7 in increments of 0.1 . (dark shading) $\left\langle\lambda_{s}\right\rangle / \overline{\lambda_{c i}}>0$; (light shading) $\left\langle\lambda_{s}\right\rangle / \overline{\lambda_{c i}}<0$. Detection points are marked by white crosses.

uniform shading everywhere other than the condition point in figure 10. The ensemble averages of figures $10(a)-10(d)$ consistently indicate that this is not in fact the case. Whilst there is some indication that the ensembles are not fully converged (residual noise in the contour fields), for all four detection heights shown a concentration of negative signed swirl (lighter shaded patch) is noted to occur on the right-hand side $(\Delta y>0)$ of the conditioned positive event. Figure 10 would seem to indicate some statistical predominance for counter-rotating vortex pairs bisecting the $135^{\circ}$ plane with a 'common-flow-up' located at the confluence. Such a counter-rotating pair is illustrated schematically over the contours of figure $10(d)$. This result can be clarified by plotting the spanwise profile of the conditionally averaged swirl at $z=z_{\text {ref }}$ (along the black dotted lines shown in figure 10a). This is shown in figure 11(a). Clearly, the swirl is asymmetric in the spanwise direction, exhibiting a consistent negative tendency at $\Delta y>0$. Indeed this trend is noted at all values of $z_{\text {ref }}$. The mean value of $\left\langle\lambda_{s}\right\rangle$ has been calculated to the left- and right-hand-side of the detection point (evaluated across the regions bounded by the dashed boxes in figure 10 and denoted by the 

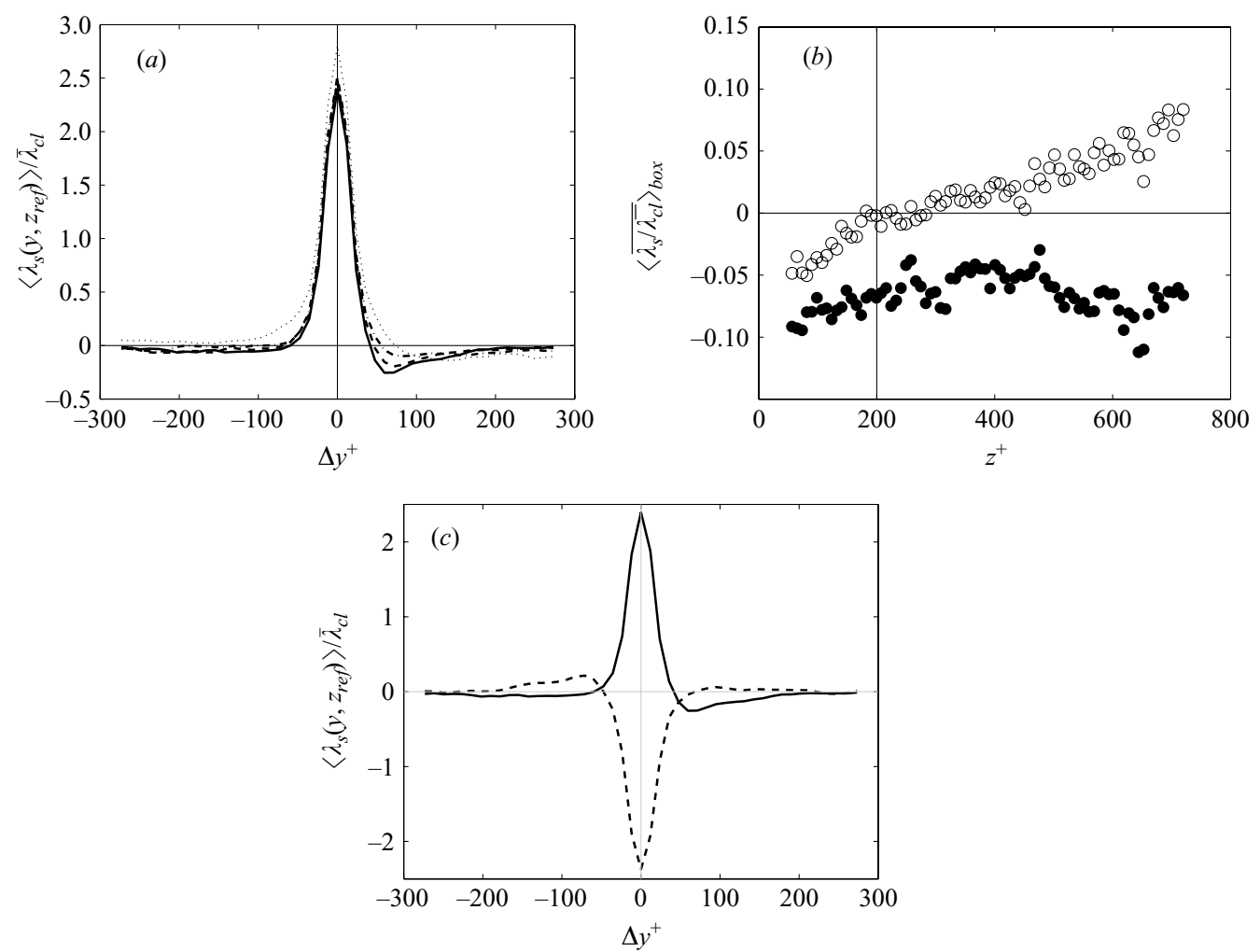

FIGURE 11. Ensemble-averaged in-plane swirl results for $135^{\circ}$ inclined plane. (a) Spanwise profile $\left\langle\lambda_{s}\left(y, z_{\text {ref }}\right)\right\rangle$ given $\lambda_{s}\left(0, z_{\text {ref }}^{\prime}>t h\right)$; (solid) $z_{\text {ref }}^{+} \approx 50$; (dashed) $z_{\text {ref }}^{+} \approx 100$; (dot-dashed) $z_{\text {ref }}^{+} \approx 150$; (dotted) $z_{\text {ref }}^{+} \approx 600$. (b) Averaged $\left\langle\lambda_{s}\right\rangle$ over; (solid) right-hand side box; (open) left-hand side box. Results are averaged within the dashed boxes marked on figure 10. (c) Spanwise profile at $z_{\text {ref }}^{+} \approx 50$; (solid) $\left\langle\lambda_{s}\left(y, z_{\text {ref }}\right)\right\rangle$ given $\lambda_{s}\left(0, z_{\text {ref }}^{\prime}>t h\right)$; (dashed) $\left\langle\lambda_{s}\left(y, z_{\text {ref }}\right)\right\rangle$ given $\lambda_{s}\left(0, z_{\text {ref }}^{\prime}<-t h\right)$.

overbar). These results are shown in figure $11(b)$ for a wide range of $z_{\text {ref }}$. Despite the scatter in the data, it is clear that the right-hand side exhibits a consistently more negative mean value. In other words, it is statistically more likely that a negative swirl will form on the positive $\Delta y$ flank of a positive swirl event. It is noted that near the wall, the left-hand side exhibits a weaker negative tendency, becoming positive at $z^{+} \approx 200(z / \delta \approx 0.2$, the approximate edge of the log region). This would imply that there is a weaker diminishing tendency throughout the log region for occasional common-flow-down vortex pairings. Regardless, the swirl value on the right-hand side is always less (more negative) that the left-hand side. This disparity in the box averaged $\left\langle\lambda_{s}\right\rangle$ becomes more pronounced towards the edge of the boundary layer.

In terms of probabilities, the lighter shaded contours to the right of the conditioned positive swirl event in figure 10, indicate an increased probability of negative swirl in these regions (over and above the background level). In the log region (at $z^{+}=100$ ) these contours indicate that a negative signed swirl event is $17 \%$ more probable (as compared to background levels). In the wake region, at $z^{+}=600(z / \delta=0.61)$, this figure rises to approximately $25 \%$. In general, the conditional swirl results for the $135^{\circ}$ plane seem to be consistent with our initial observations from the example instantaneous swirl fields of figure $6(f)$, where there was an observed tendency for 

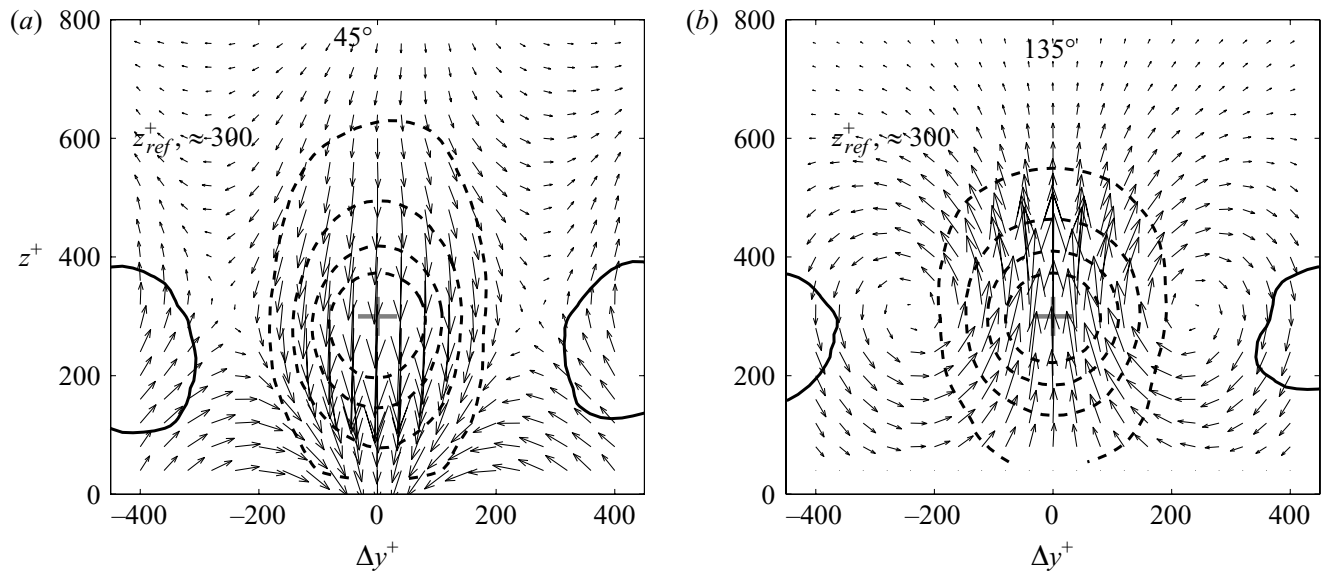

FiguRE 12. A single-point linear stochastic estimate on the presence of a low-momentum region $\left(u<-4 U_{\tau}\right)$ at $\left(0, z_{\text {ref }}=300\right)$. (a) $45^{\circ}$ case; (b) $135^{\circ}$ case (for $\left.R e_{\tau}=1010\right)$. Contours show conditioned streamwise velocity (contour levels $\langle u\rangle=-3 U_{\tau}$ to $0.75 U_{\tau}$ ) increments of $0.75 U_{\tau}$ (negative contours are dashed). In-plane $\left(v_{\theta}\right.$ and $\left.w_{\theta}\right)$ velocity vectors are shown in black. Vector grid spacing is down-sampled for clarity of presentation.

positive and negative swirls to align to the left and right, respectively, of the low-speed ejection labelled I. They also mesh well with the hairpin-packet scenario. Certainly, the model sketched in figure 9 would indicate that hairpin packets lead to a predominance of common-flow-up counter-rotating swirl pairings in the $135^{\circ}$ plane. However, the fact that these percentages are so low indicates that the vortical structure retains a certain degree of asymmetry. From low-Reynolds-number DNS data, Robinson $(1990,1991)$ noted that vortical arches are often 'lop-sided' and that near-wall quasistreamwise vortices 'occur only rarely as equal-strength counter-rotating pairs'. As a further validation and a check for homogeneity, Figure 11(c) shows the same average performed on a negative signed conditioning event at $z_{\text {ref }}^{+}=50$. As would be expected, this shows the opposite scenario to the conditioned positive swirl. There is an increased probability of a positive swirl event occurring to the left (at $-\Delta y$ ) of the condition point.

\subsection{Detection on low streamwise momentum}

Low-speed regions, of the type highlighted by the dashed contours in figure 6, are here investigated statistically. Linear stochastic estimation (Adrian \& Spalart 1988) is used to estimate the conditional velocity field on the presence of a negative streamwise velocity event at the detection height $z_{\text {ref }}$ (written as $\left\langle u_{i}(\Delta y, z) \mid u_{1}\left(0, z_{\text {ref }}\right)\right\rangle$ ). In this case, where the detection event is defined by a single velocity component, the linear estimate has been shown (e.g. Tomkins \& Adrian 2003) to reduce to

$$
\left\langle u_{i}(\Delta y, z) \mid u_{1}\left(0, z_{\text {ref }}\right)\right\rangle=L_{i 1} u_{1}\left(0, z_{\text {ref }}\right),
$$

where

$$
L_{i 1}=\frac{\left\langle u_{1}\left(0, z_{r e f}\right) u_{i}(\Delta y, z)\right\rangle}{\left\langle u_{1}^{2}\right\rangle} .
$$

Thus for this simple detection event, the linear stochastic estimate (LSE) is merely a function of the two-point cross-correlations $R_{u u}, R_{u v}$ and $R_{u w}$ calculated on unconditional data. The resulting estimate at $z_{\text {ref }}^{+}=300$ is shown in figure 12 . The detection criteria dictates that there will always be a low-speed region at $\left(0, z_{\text {ref }}\right)$. 


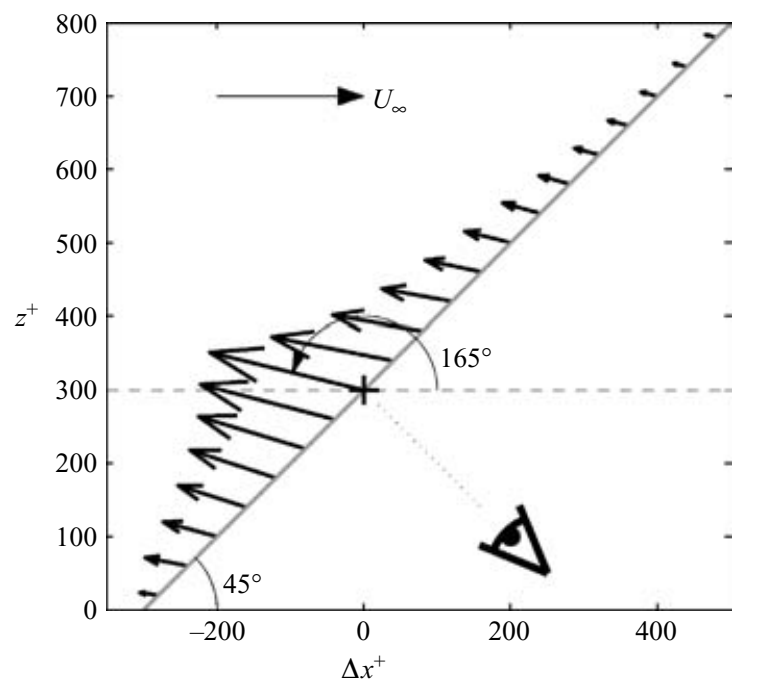

FIGURE 13. Side-view of the $45^{\circ}$ single-point linear stochastic estimate shown in figure $12(a)$. Vectors displayed at $\Delta y=0$ to show the calculated ejection angle.

Contours of $\langle u\rangle$ show that this event is bordered by a high-speed region at $\Delta y^{+} \approx \pm 400$ in both planes. The wall-normal extent of the low-momentum region is consistently greater for the $45^{\circ}$ plane than for the $135^{\circ}$ case (for all $z_{\text {ref }}$ ). This reflects the structural inclination angle associated with both the individual hairpin vortices, and the ramp-like packet arrangements. Ganapathisubramani et al. (2005) noted a similar inclined tendency for the same inclined data set. Clearly, the hairpin packet scenario (with the characteristic growth angle), as depicted in figure 9, would lead to a taller low-momentum region in the $45^{\circ}$ inclined plane than for the $135^{\circ}$ case.

Considering the velocity vectors in figure 12 , the $135^{\circ}$ LSE result clearly shows in-plane swirling motions. Such a swirl is almost entirely lacking from the $45^{\circ}$ plane (which exhibits a saddle-like flow pattern). The disparity in swirl between the two planes is consistent with the results of figures 6 and 7, and also the subsequent BiotSavart calculations of figure 8, all of which pointed towards an inclined structure as the source for this imbalance. In the $135^{\circ}$ plane, there is a familiar pattern of ejection, with a counter-rotating vortex pair accompanying the conditioned low-speed event. A preference for such counter-rotating pairings was found in the conditioned swirl results of figure 10 (and also predicted from the hairpin packet model of figure 9). The vectors in the $45^{\circ}$ plane of figure $12(a)$ are more difficult to interpret. These are in-plane velocity vectors, measured on inclined planes, and it is incorrect to interpret vectors pointing up or down the page as implying motion towards or away from the wall. Though not immediately obvious, the vectors pointing down the page at the condition point of figure 12(a) actually result from an ejection of low-speed fluid. Figure 13 clarifies this point by showing a side view of the conditioned event in the $45^{\circ}$ plane (at $\Delta y=0$ ). The ejection angle at the condition point is greater than $135^{\circ}$ (actually $165^{\circ}$ ), and thus when these vectors are projected into the $45^{\circ}$ plane (i.e. viewing from a point perpendicular to the image plane) they will appear as a negative $w_{45}$ event (as seen in figure 12a). The converging saddle-flow pattern of streamlines shown in figure $12(a)$ is similar to that found by Perry \& Chong (1994) when they took a slice through a Burgers vortex undergoing axisymmetric shearing and at a 

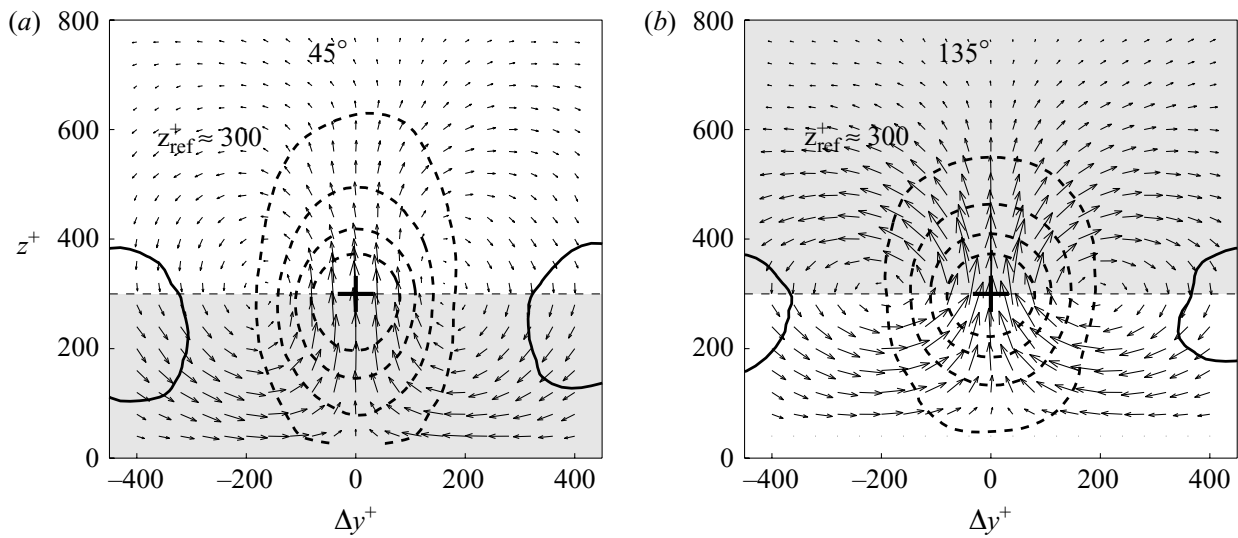

FIGURE 14. As figure 12 but with spanwise $\langle v\rangle$ and wall-normal $\langle w\rangle$ velocity vectors. White, downstream; shaded, upstream.

large oblique angle to the measurement plane. This is essentially the scenario revealed by the LSE.

Figure 14 shows the same LSE result as figure 12, with the velocity components transformed from inclined axes back to a conventional streamwise, spanwise and wall-normal system. Viewed in this way (as if viewing in the negative $x$ direction of figure 13), there is a clear $v-w$ swirling motion associated with the conditioned event in both planes. The induced components are stronger in the $135^{\circ}$ plane, which exhibits a stronger and more compact core. When viewing such figures, it is important to recall, that for the $45^{\circ}$ case, points below the detection event are actually located upstream of the origin (if $z<z_{\text {ref }}$, then $\Delta x<0$ ). Conversely, the flow field above $z_{\text {ref }}$ will be increasingly downstream of detection. The opposite is true for the $135^{\circ}$ plane.

We can understand the flow geometry associated with the LSE event better by viewing both planes simultaneously in three dimensions. Figure 15(a) shows such a construct. Since both planes are conditioned on the same event, and with the same condition point $\left(z_{r e f}\right)$, the linear stochastic estimates of figure 14 can be stacked together, with the intercept located at $\left(\Delta x=0, \Delta y=0, z=z_{\text {ref }}\right)$. The colour scaling on figure 15 shows the strength and sign of the spanwise velocity component. The $v-w$ flow fields in both inclined planes are entirely consistent, meshing well at the intercept. It is noted that the flow pattern can be explained reasonably by a pair of counter-rotating vortices inclined at an angle somewhat less than $45^{\circ}$ to the $x$-axis. This idealized structure is sketched in figure $15(a)$. Such an arrangement can explain the weaker more diffuse spanwise components in the $45^{\circ}$ plane. Figures $15(b)$ to $15(e)$ help clarify the inclined nature of the structure. They show spanwise induced velocities in $45^{\circ}$ and $135^{\circ}$ planes owing to a counter-rotating vortex pair at various inclination angles $\alpha$ (calculated using the Biot-Savart law). If the conditional eddy is at $0^{\circ} \dagger$ (figure $15 b$ ) we might expect the induced spanwise velocities to be comparable in both the forward and aft planes. Conversely, if the conditional eddy has a mean inclination angle exactly equal to $45^{\circ}$ (figure $15 d$ ), there would be zero ensembleaveraged spanwise motion in the $45^{\circ}$ plane. Between these two extremes, with an inclination angle less than $45^{\circ}$ (figure $15 c$ ), we see more diffuse spanwise motions

$\dagger$ Although in reality it is recognized that a pure streamwise vortex is paradoxical in the presence of a mean wall-normal shear. 

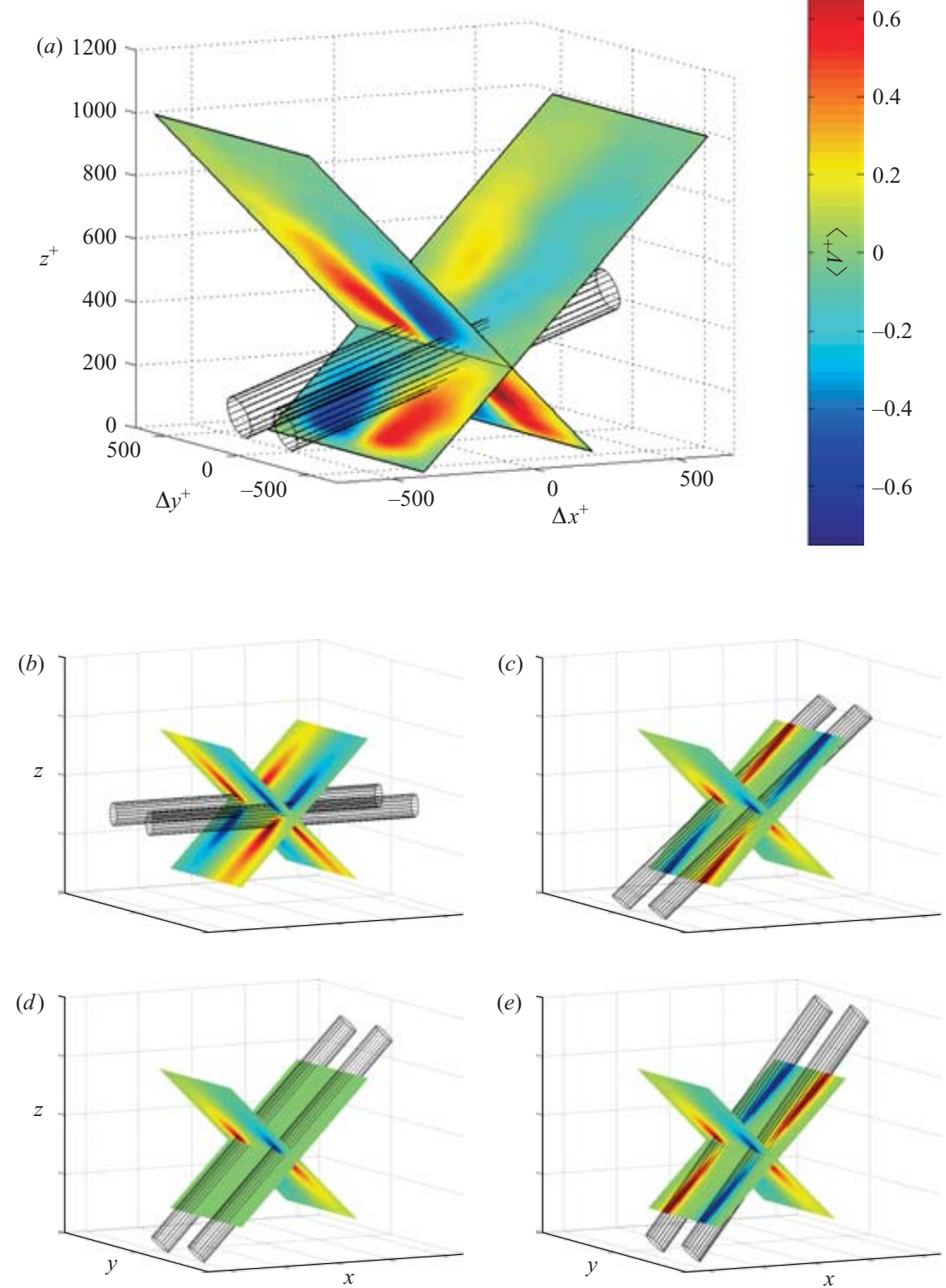

FIGURE 15. (a) Three-dimensional flow geometry of the LSE events shown in figure 14. Colour shading shows conditioned spanwise velocity $\left\langle v^{+}\right\rangle$. A schematic representation of an idealized conditional eddy is included. Biot-Savart calculated $\langle v\rangle$ due to an inclined counter-rotating vortex pair inclined at (b) $\alpha=0 ;(c) \alpha<45^{\circ} ;(d) \alpha=45^{\circ} ;(e) \alpha>45^{\circ}$.

in the $45^{\circ}$ plane. Finally, if the inclination angle was consistently greater than $45^{\circ}$ (figure $15 e$ ), there would be opposite signed spanwise velocity in the $45^{\circ}$ plane (red regions where there are blue in $135^{\circ}$ plane and vice versa). Clearly, figure $15(a)$ lies somewhere closer to the scenario shown in figure $15(c)$, with the proviso that the LSE event generally weakens as we move further from the condition point. The actual 

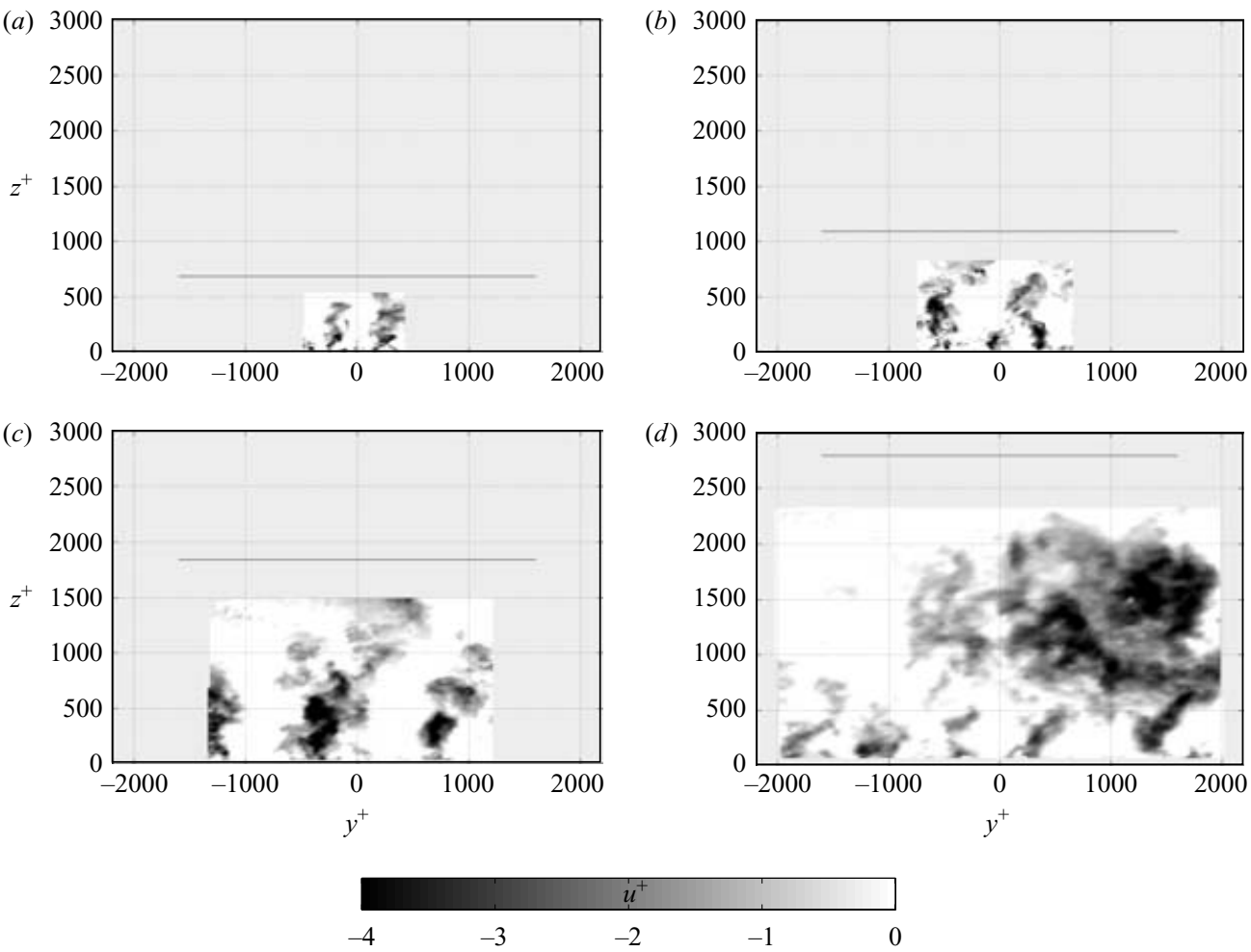

FIGURE 16. Contours of negative streamwise velocity fluctuation $\left(u^{+}<0\right)$ for $135^{\circ}$ plane; (a) $R e_{\tau}=690 ;$ (b) $R e_{\tau}=1010 ;$ (c) $R e_{\tau}=1840 ;$ (d) $R e_{\tau}=2800$. Darker shading indicates larger negative fluctuations. All positive $u$ fluctuations are shaded white. Solid horizontal line shows $\delta$.

angle of the structure in figures $15(b)$ to $15(e)$ can be recovered from the angle made by the maxima and minima of the induced $\langle v\rangle$ component in the two planes. If we apply this criteria to the LSE event of figure $15(a)$, the spanwise induced velocities indicate a conditional eddy inclination angle of $\alpha \approx 20^{\circ}$. However, this is an angle for a conditioned event and does not necessarily indicate the inclination angles of individual instantaneous hairpin structures. It must always be remembered that the LSE event is essentially based on two-point correlation results. Such correlations will reflect a multitude of scales and events, and in particular we would expect such angles to be contaminated by the packet inclination angle (see figure 9 and Ganapathisubramani et al. 2005).

\section{Reynolds number scaling}

Thus far we have dealt almost exclusively with the $R e_{\tau}=1010$ data set. By comparing two-point correlations at all four $R e_{\tau}$, the following analysis will consider Reynolds-number scaling of the dominant flow structures.

\subsection{Spanwise length scales}

Before presenting correlation results, let us briefly consider an example instantaneous streamwise flow field for each of the four Reynolds numbers, as shown in figure 16. The figures are plotted on inner scaled axes $\left(y^{+}\right.$and $\left.z^{+}\right)$demonstrating that, in terms 

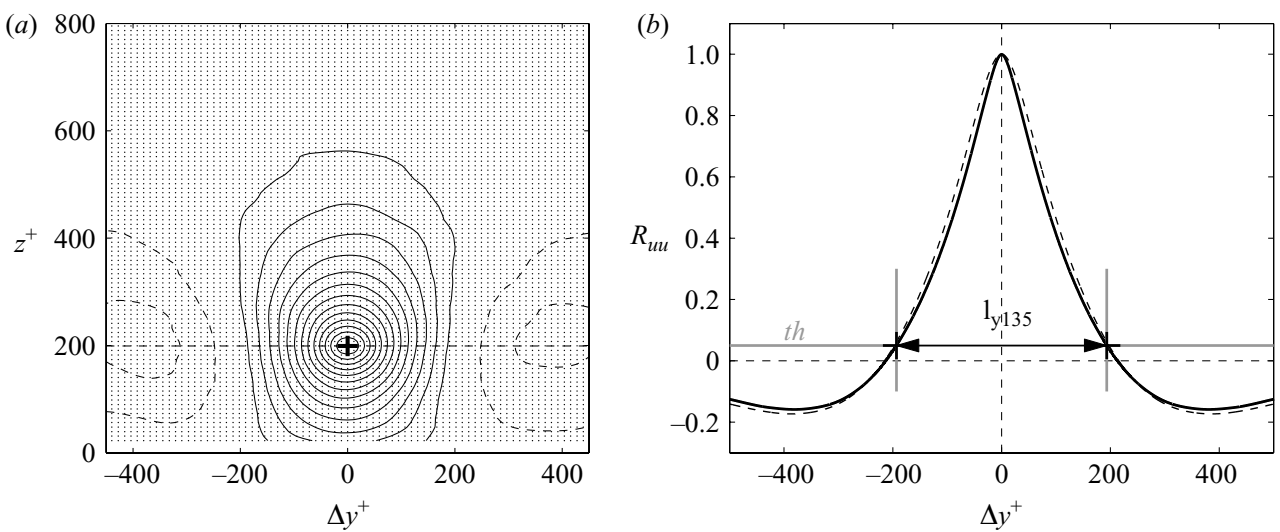

FiguRE 17. Example two-point correlation results $R_{u u}$ for $135^{\circ}$ plane $\left(R e_{\tau}=1010\right)$ with $z_{\text {ref }} \approx 200 ;(a)$ contours of $R_{u u}(\Delta y, z)$, contour levels from -0.14 to 0.98 in increments of 0.07 . Negative levels shown as dashed contours. Grid indicates vector resolution; $(b)$ plot of $R_{u u}\left(\Delta y, z_{\text {ref }}\right)$ showing definition of a characteristic spanwise length scale determined on the intercept with some given threshold $t h$ (in this case $t h=0.05$ ). Dashed lines show the same result from images reprocessed using a $64 \times 64$ interrogation window.

of viscous wall units, the relative viewing window increases dramatically across the tested Reynolds number range (hence the reduction in resolution). The shading on these figures shows only regions of retarded streamwise velocity $\left(u^{+}<0\right)$. For these frames, taken at an inclination angle of $135^{\circ}$, a predominance of tall (stripy) low-speed regions are noted, erupting a considerable distance into the boundary layer from the wall. In general, between each of these low-speed strips there exists a corresponding region of accelerated flow, with swirling motions occurring at the high shear confluence between high- and low-speed zones. Such flow patterns were previously highlighted by the region labelled I in figure $6(b, d, f)$, and shown to be typical of the hairpin packet paradigm of figure 9. At the highest Reynolds number (figure $16 d$ ), there is some indication of possible hierarchical distributions, with a sudden jump in scale occurring at $z^{+} \gtrsim 500-600$. This phenomena will be touched upon later. However, the main point to take from the figure, is that viscous scaling is clearly inadequate to describe the Reynolds-number development of the characteristic spanwise length scale. In terms of $y^{+}$, even close to the lower limit of the log region, the spanwise length scale for high- and low-speed regions seems to increase dramatically with Reynolds number. This observation can be confirmed with two-point correlations.

Figure 17 illustrates the process by which a characteristic length scale is extracted from a two-point correlation. In this instance, the spanwise length scale is extracted from $R_{u u}$ at $R e_{\tau}=1010$ for the $135^{\circ}$ case. Figure $17(a)$ shows contours of $R_{u u}$ for $z_{\text {ref }}=200$. This is typical of any $R_{u u}$ correlation for the inclined planes in the $\log$ region. A central region of positive correlation, elongated in the wall-normal direction, is flanked by anti-correlated lobes (in this case $R_{u u}$ seems to switch signs at $\left.\Delta y^{+} \approx \pm 200\right)$. Such behaviour is consistent with the tendency for alternating regions of high- and low-speed noted previously in figure 16. Hutchins et al. (2004) and Ganapathisubramani et al. (2005) have shown that these large-scale correlated and anti-correlated regions in the inclined planes actually persist for a considerable distance in the streamwise direction. Figure $17(b)$ takes a slice through this contour map at $z=z_{\text {ref }}$ (along the horizontal line in figure 17a). The dashed curve in figure $17(b)$ indicates the same two-point correlation calculated from vector fields 

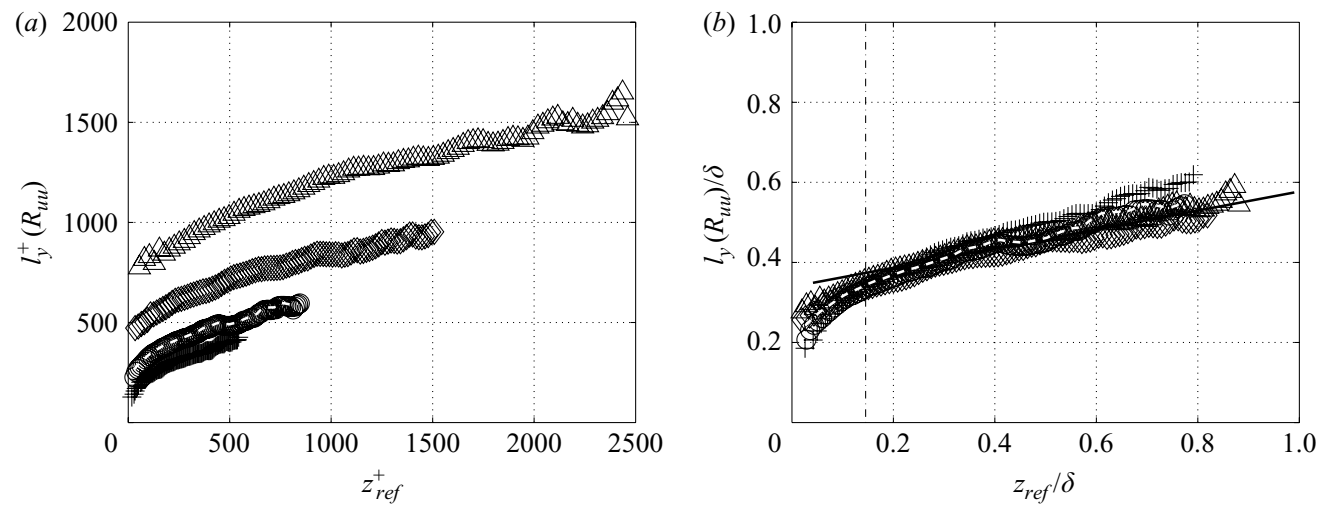

FIGURE 18. Variation with $z_{\text {ref }}$ of the characteristic spanwise length scale (based on streamwise velocity correlations $R_{u и}$ ) for $135^{\circ}$ plane; $(a)$ inner scaling; $(b)$ outer scaling. th $=0.05$. $\times$, $R e_{\tau}=690 ; \bigcirc, R e_{\tau}=1010 ; \diamond, R e_{\tau}=1840 ; \triangle, R e_{\tau}=2800$. Dashed line shows $R e_{\tau}=1010$ data reprocessed with $64 \times 64$ interrogation window. Dash-dot line on $(b)$ shows $z^{+}=100$ at $R e_{\tau}=690$.

that have been resolved using a $64 \times 64$ pixel interrogation window $\left(i^{+}=48\right)$. This will filter the smaller scales, and is included to simulate the effects of the larger $i^{+}$ necessary for the $R e_{\tau}=1840$ and $R e_{\tau}=2080$ experiments. A comparison of the dashed and solid lines in figure $17(b)$ reveals that such a filter has only a marginal effect on the correlation results. As would be expected, any difference is most noticeable at smaller values of $\Delta y$ where the two-point correlation is largely driven by smallscale phenomena. For the most part, we will be unconcerned by this region of the correlation map since we are looking for a length scale that can capture the larger-scale structure (signatures of the hairpin/hairpin packet), at which point the differences between the $32 \times 32$ and the $64 \times 64$ pixel $R_{u u}$ results are insignificant. Spanwise length scales are extracted by establishing some nominal threshold (in this case $t h=0.05$ ), and finding the intercept of $R_{u u}$ with this threshold. This process is repeated for all wall-normal locations $\left(z_{\text {ref }}\right)$, for both planes and at all Reynolds numbers. Figure 18 shows the spanwise length scales based on $R_{u u}$ (written as $l_{y}\left(R_{u u}\right)$ ) for the $135^{\circ}$ inclined plane. Figure $18(a)$ is presented on inner scaled axes and reaffirms the initial observation from figure 16 (namely that viscous scaling is not the correct way to non-dimensionalize the spanwise length scale). When scaled using boundary-layer thickness $\delta$ (figure 18b), the collapse is convincing for all four Reynolds numbers tested in the logarithmic and outer regions. For the majority of the boundary layer, the $R_{u и}$ spanwise length scale exhibits a consistent approximately linear increase with $z_{\text {ref }}$, although there are signs that this increase is more pronounced in the log region (in approximately the first fifth of the boundary layer). A similar change in the $l_{y}\left(R_{u u}\right)$ growth rate was noted by Ganapathisubramani et al. (2005) in a comparison between inclined plane data and previously obtained spanwise/streamwise PIV. In figure 18(b), there appears to be a peal-off near the wall from the collapsed curve, which is especially noticeable for the two lowest Reynolds numbers. This is expected since the first wall-normal stations for these flows are not in the log region $\left(z^{+}<100\right)$ and hence outer scaling of $R_{u u}$ would no longer be expected to apply. The dash-dot line in figure $18(b)$ indicates $z^{+}=100$ for the lowest Reynolds number. It is clear from the white dashed lines in figure 18 , that reprocessing the $R e_{\tau}=1010$ images using a $64 \times 64$ pixel spot size in no way influences the conclusions. 

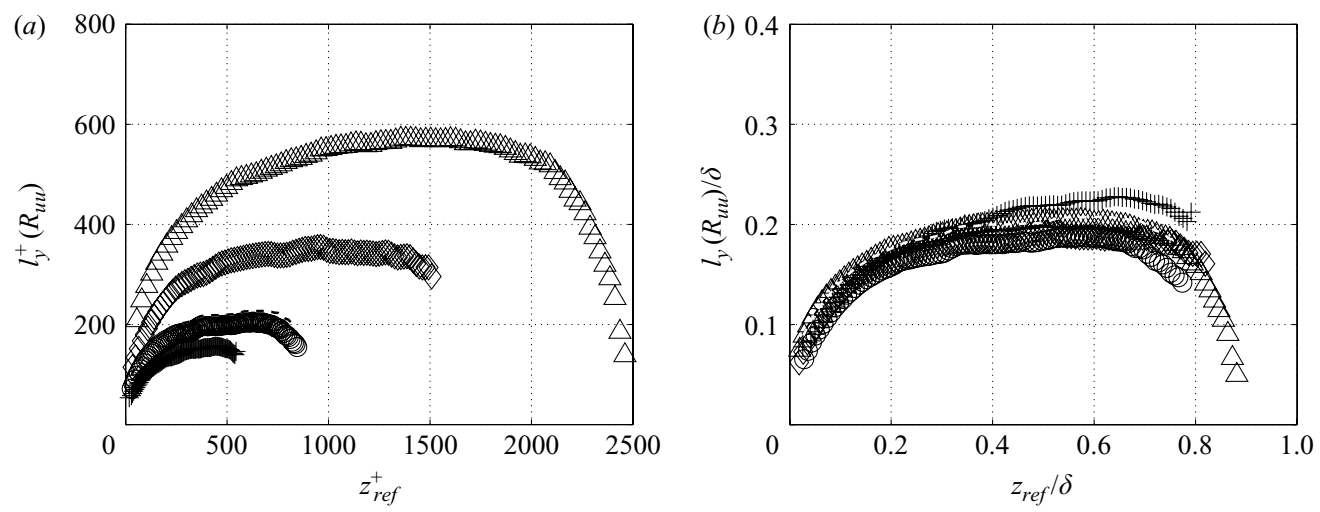

FIGURE 19. As figure 18 except with increased threshold $(t h=0.5)$.

The exact profiles of the collapsed outer-scaled curves are very much dependent on the threshold level chosen to extract $l_{y}\left(R_{u u}\right)$. However, the salient trends are the same. Even if th is set as high as 0.5, there is still no collapse in terms of viscous scaling, whilst the $\delta$-scaled results exhibit reasonable collapse. Such results are shown in figure 19. In this case, the disparity caused by the increased spot size (dashed line) is larger, leading to an approximate $10 \%$ shift in $l_{y}\left(R_{u u}\right)$. However, even this error is well within the spread of the 'collapse' and would in no way alter the principle conclusions regarding outer scaling.

For completeness, spanwise length scales are extracted by similar techniques for the spanwise and wall-normal velocity correlations $\left(R_{v v}\right.$ and $\left.R_{w w}\right)$. These results are presented in figure 20. It is again clear that for the range of $R e_{\tau}$ tested, $R_{v v}$ and $R_{w w}$ both also scale with outer variables across the entire region of measurement. In figure $20(d)$ there is a marked increase in the spanwise width of the wall-normal correlations occurring at approximately $z_{\text {ref }} / \delta \approx 0.6$. The precise reasons for this are somewhat unclear, although it appears to be caused by the disappearance of any flanking anti-correlated region (such as that evident for $R_{u u}$ in figure $17 \mathrm{~b}$ ), without which the positive correlation peak exhibits a more gradual decay from the reference point. The physical significance of this is difficult to isolate, although the spanwise width of the $R_{w w}$ contours will to some degree include a measure of the vortex core diameter. The rapid increase in $l_{y}\left(R_{w w}\right)$ occurs at a wall distance where $\omega_{x^{\prime}}$ and $\lambda_{c i}$ statistics would indicate that vortex cores are becoming rare (see figure 7 ).

The results in figure 18 and 19 are consistent with previously published results, such as Wark, Naguib \& Robinson (1991) who noted that 'the structures in the log region seem to scale with outer variables over the entire range of spanwise scales.' Similarly, Mclean (1990) concluded, for $y^{+} \gtrsim 40$, that the spanwise and streamwise correlations (and hence length scales) of $R_{u u}$ scaled with outer variables. The approximately linear growth of spanwise length scales with $z$ as shown in figure 18 is also consistent with the proposed scaling in the attached eddy hypothesis (Townsend 1956; Perry \& Chong 1982; Marusic \& Perry 1995).

It is useful to consider briefly the actual structure in the $\log$ and wake regions that has lead to the outer scaled length scales under discussion here. The log region is dominated by long streamwise aligned regions of alternating high and low speed $u$ (with a width of approximately $0.4 \delta$, similar to that suggested by figure $18 a$ ). Instantaneous examples of these features are given by Ganapathisubramani et al. (2003), Tomkins \& Adrian (2003) and Hutchins, Ganapathisubramani \& Marusic 

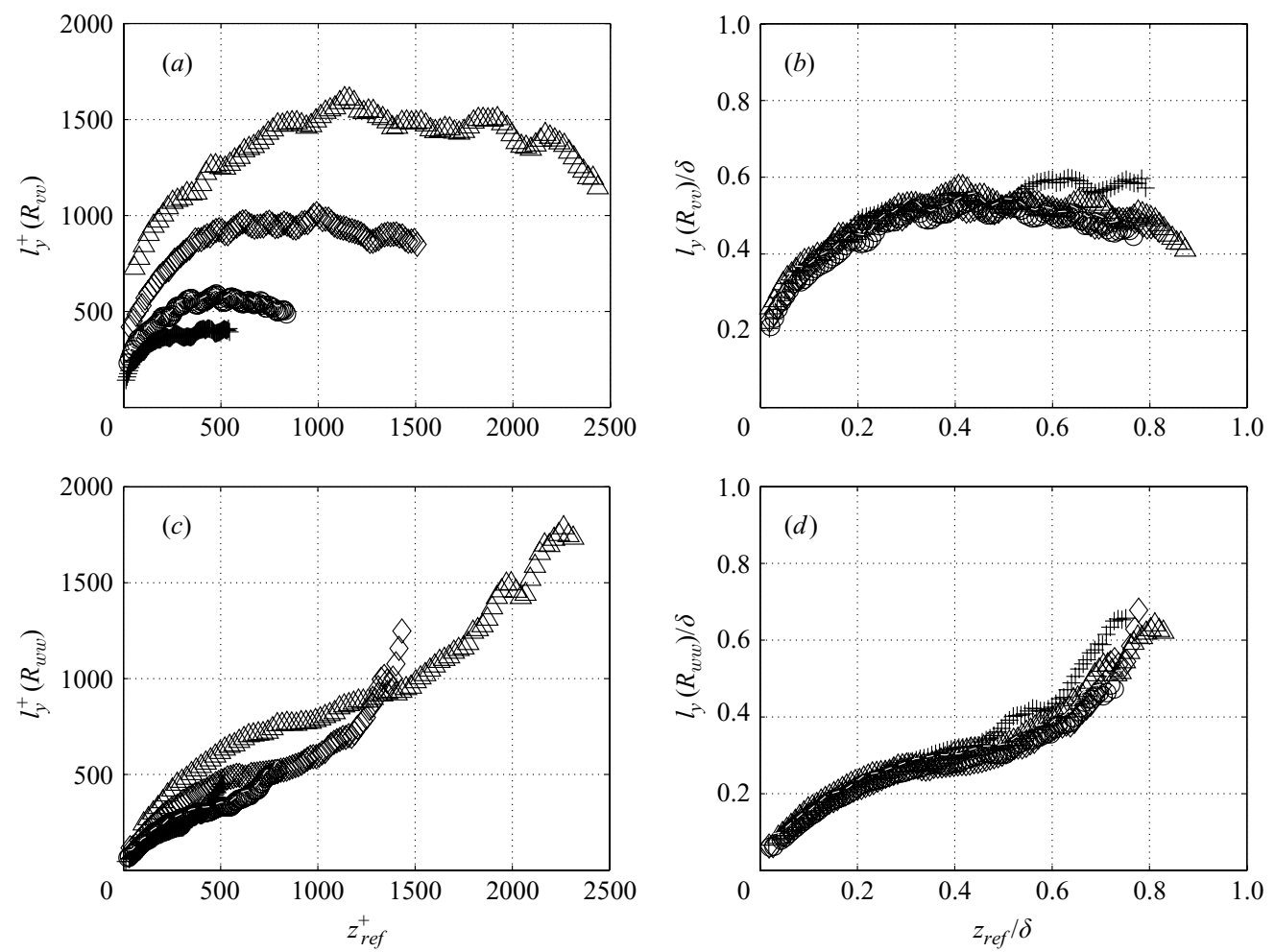

FIGURE 20. Variation with $z_{\text {ref }}$ of the characteristic spanwise length scale based on (upper) spanwise correlations $R_{v v}$; (lower) wall-normal correlations $R_{w w}$ in the $135^{\circ}$ plane; $(a) l_{y}\left(R_{v v}\right)$ inner scaling; $(b) l_{y}\left(R_{v v}\right)$ outer scaling; $(c) l_{y}\left(R_{w w}\right)$ inner scaling; $(d) l_{y}\left(R_{w w}\right)$ outer scaling. $t h=0.05$ for $l_{y}\left(R_{v v}\right)$ and $t h=0.15$ for $l_{y}\left(R_{w w}\right)$. Dashed line shows $R e_{\tau}=1010$ data reprocessed with $64 \times 64$ interrogation window. $\times, R e_{\tau}=690 ; \bigcirc, R e_{\tau}=1010 ; \diamond, R e_{\tau}=1840 ; \triangle, R e_{\tau}=2800$.

(2004). Two-point correlations in the spanwise/streamwise plane are presented in Tomkins \& Adrian (2003) and Ganapathisubramani et al. (2005). These long regions of streamwise momentum fluctuation are believed to be the footprint of packet-type structures (specifically, the long low-speed regions are thought to be caused by an induced momentum deficit between the legs of hairpin vortices advecting in a packet arrangement). Tomkins \& Adrian (2003) and Ganapathisubramani et al. (2003) have shown that vortex cores align at the shear layer between these high- and low-speed regions in an arrangement that is consistent with the packet model. In many of these cited studies, the length of such features exceeds the PIV frame. Kim \& Adrian (1999) have reported very long packet-type structures in fully developed pipe flows and Hutchins et al. (2004) have reported similarly long meandering features in the log region of turbulent boundary layers (often exceeding $20 \delta$ in length). Large outerscaled coherence is also noted in recent DNS results performed at $R e_{\tau}=950$ (del Alamo et al. 2004), both in the available instantaneous flow fields and in the twodimensional energy spectra (del Álamo \& Jiménez 2003). In short, the underlying implication to the preceding scaling arguments is that, depending on one's precise viewpoint, the 'packet structure' (Adrian and coworkers), 'superburst' (Hanratty and coworkers) or 'large-scale passive wakes' (Jiménez \& del Álamo 2004) all scale with boundary-layer thickness. 

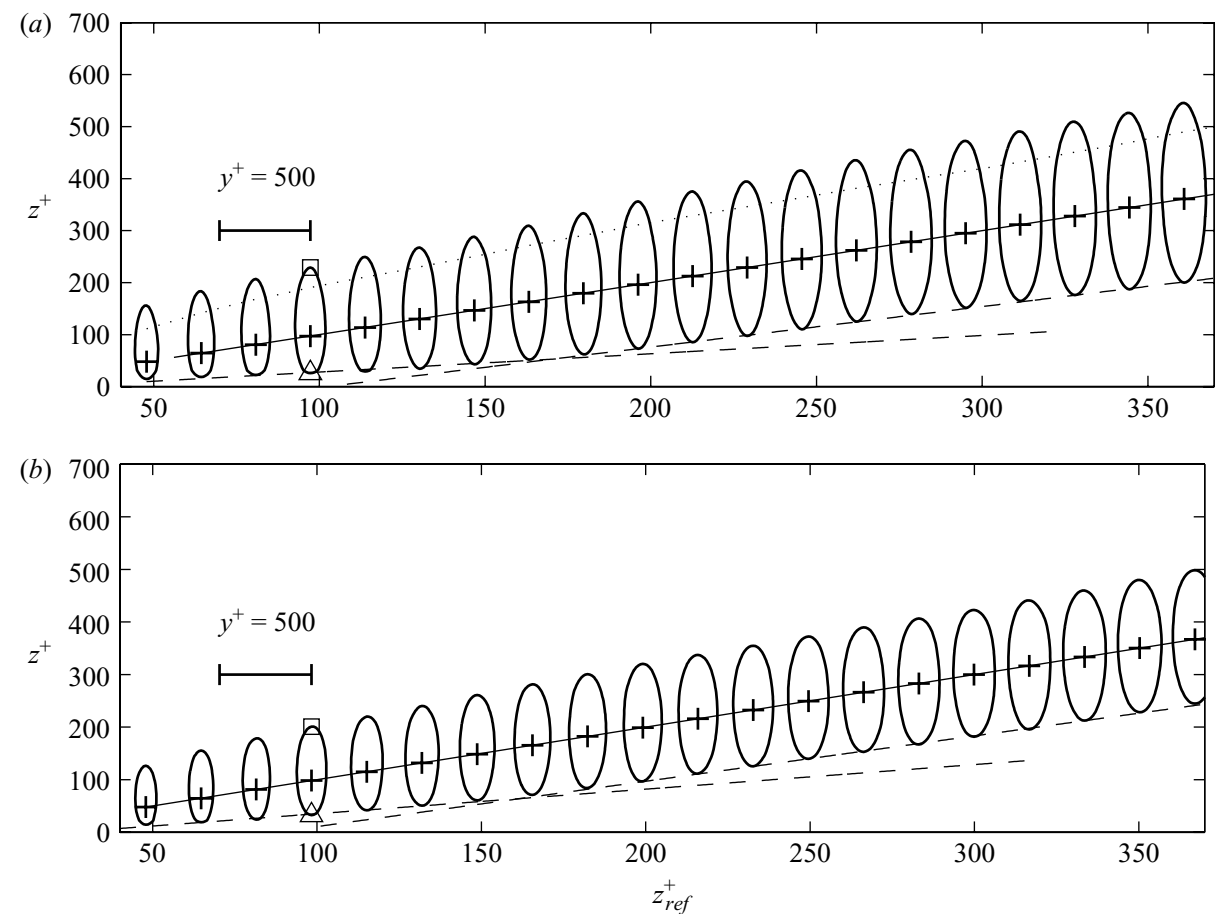

FIGURE 21. Attached and detached correlation regimes for $R e_{\tau}=1010$ data set. $R_{u и}=0.4$ contours are plotted for increasing $z_{\text {ref }}$ across the boundary layer; $(a) 45^{\circ}$ plane; $(b) 135^{\circ}$ plane. For clarity, contours are only shown at every other available wall-normal station. Maximum and minimum wall-normal extent of contours are shown by: $\square, z_{c \theta(\max )} ; \Delta, z_{c \theta(\min )}$; ,$+ z_{\text {ref }}$ location for each contour; - -, linear fit to $z_{c \theta(\min )}$. The dotted line in plot $(a)$ shows the maximum wall-normal extent of corresponding contours in the $135^{\circ}$ plane.

\subsection{Wall-normal length scales: 'attached' and 'detached' events}

The PIV results can also be used to extract some characteristic wall-normal length scale from the two-point correlations. Up to certain values of $z_{\text {ref }}$, it is found that the wall-normal (or in this case $z^{\prime}$ ) extent of a given $R_{u u}$ contour, appears to be influenced by the wall. The example correlations of figure $17(a)$ give a good early indication of this behaviour, with the largest solid contours exhibiting asymmetry about $z_{\text {ref }}$, giving the impression that they have been curtailed by (or 'splatted' on to) the wall. To illustrate this behaviour in more detail, an $R_{u u}$ contour is extracted and plotted for each available $z_{\text {ref }}$ across the boundary layer. These are shown in figure 21 for both the $45^{\circ}$ and the $135^{\circ}$ cases (with $R_{u u}=0.4$ ). For a given $z_{\text {ref }}$, the contours extend further in the wall-normal direction for the $45^{\circ}$ than for the $135^{\circ}$ plane. The dotted line drawn over the $45^{\circ}$ contours of figure $21(a)$, show the maximum wall-normal extent of the corresponding contour in the $135^{\circ}$ plane. Such disparity is symptomatic of the inclined nature of the large-scale structures (see $\S 5.2$ and Ganapathisubramani et al. 2005). Because of this inclined tendency, the 'curtailment' or 'splatting' of the contours is more noticeable for the $45^{\circ}$ inclination. The maximum and minimum $z^{+}$ ordinate has been extracted for each of the contours shown in figure 21 . We define these ordinates as $z_{c \theta(\max )}$ and $z_{c \theta \text { (min) }}$, and examples are marked on the $z_{\text {ref }}^{+} \approx 100$ contours of figure 21 by the square and triangular symbols, respectively. For both the $45^{\circ}$ and $135^{\circ}$ cases, there is a clear height $\left(z_{\text {ref }}\right)$ at which the contours seem to 'lift-off' 

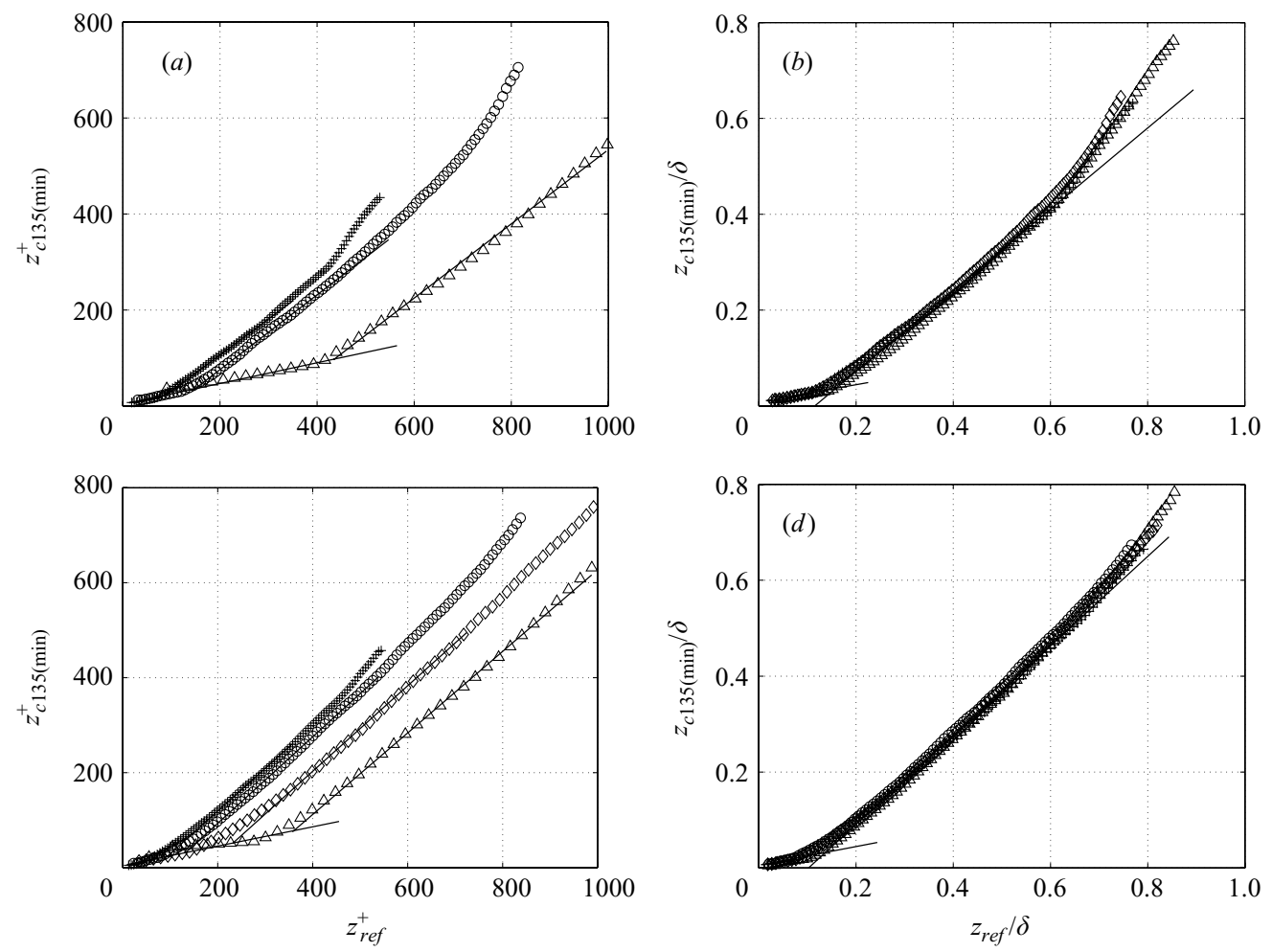

FIGURE 22. Variation of $z_{c \theta(\min )}$ with $z_{r e f}$ (calculated on $\left.R_{u u}=0.4\right) ;(a)$ inner-scaling $45^{\circ} ;(b)$ outer-scaling $45^{\circ} ;(c)$ inner-scaling $135^{\circ} ;(d)$ outer-scaling $135^{\circ} . \times, \operatorname{Re}_{\tau}=690 ; \bigcirc, \operatorname{Re}_{\tau}=1010$; $\diamond, R e_{\tau}=1840 ; \triangle, R e_{\tau}=2800$.

from the wall (or at least cease to extend as far towards it). To highlight this feature, lines (shown dashed) are fitted to $z_{c \theta(\min )}$ either side of the juncture. In this way, two distinct regimes seem to reveal themselves, with a rapid and pronounced change in $\partial z_{c \theta(\text { min })} / \partial z_{\text {ref }}$ occurring somewhere close to $z_{\text {ref }} \approx 170$. We tentatively refer to these two regimes as attached (events at $z_{\text {ref }}$ are still strongly influencing or influenced by the wall) and detached (a noticeable decorrelation between fluctuations at $z_{\text {ref }}$ and those at the wall).

The height at which detachment occurs is very much specific to the chosen $R_{u u}$ contour. However, it would be useful to determine a valid scaling argument for this phenomena. The lowermost extent of the $R_{u u}=0.4$ contour $\left(z_{c \theta(\min )}\right)$ is found at all four Reynolds numbers for both inclined planes, the results are collated in figure 22 . Figures $22(a)$ and $22(b)$ show the $45^{\circ}$ data (viscous and outer-scaled, respectively), whilst results for the $135^{\circ}$ inclination are shown in figures $22(c)$ and $22(d)$. In terms of inner-scaling (figure $22 a, c$ ), it is noted that whilst the contours remain attached, the location of the contour minima $z_{c \theta(\min )}^{+}$appear to collapse onto a common curve. However, beyond detachment there is clear evidence of Reynolds-number dependence. In contrast, the collapse of $z_{c \theta(\min )}$ with outer-scaling (figure $22 b, d$ ) is more complete. For this particular contour level, a tightly collapsed detachment point occurs at $z_{\text {ref }} / \delta_{c} \approx 0.15$ for all Reynolds numbers, with the data collapsing to two approximately linear regimes either side of this point. This would imply that outer-scaling is the correct way to characterize this phenomenon. 

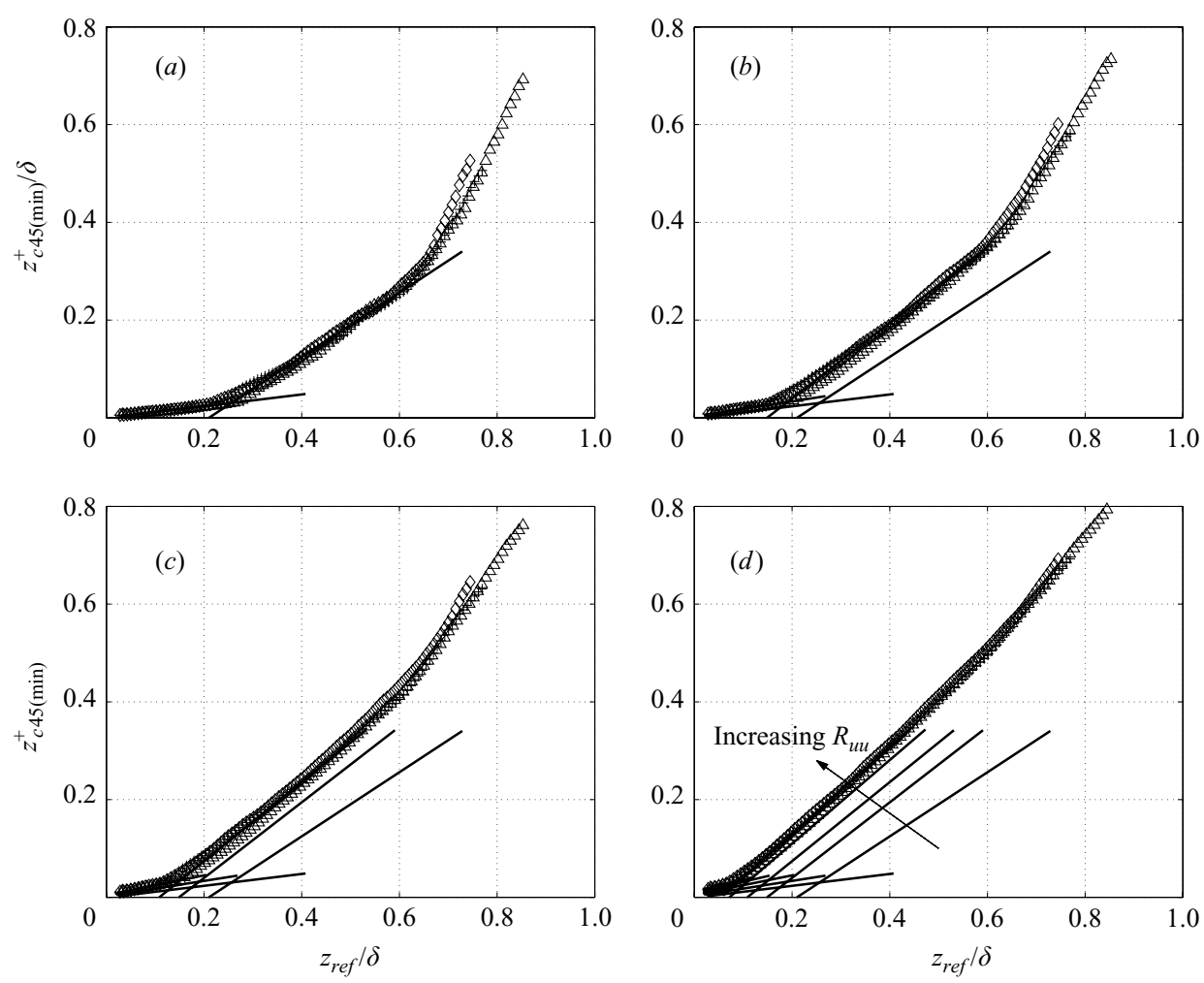

FIGURE 23. Outer-scaled $z_{c 45(\min )}$ profiles for; $(a) R_{u u}=0.2 ;(b) R_{u u}=0.3 ;(c) R_{u u}=0.4$; (d) $R_{u u}=0.6 ; \times, R e_{\tau}=690 ; \bigcirc, R e_{\tau}=1010 ; \diamond, R e_{\tau}=1840 ; \triangle, R e_{\tau}=2800$.

Figure 23 confirms the occurrence of the attached/detached contour regime across a range of contour levels. In accordance with the findings of figure 22, the results are scaled with outer-variables. At all contour levels this scaling gives good collapse with Reynolds number. Furthermore, the previously noted attached and detached behaviour is seen to occur regardless of contour level, although the precise $z_{\text {ref }}$ at which a given contour separates or detaches from the wall will be very much dependent on the chosen value of $R_{u u}$. The detachment point shifts from $z_{\text {ref }} / \delta_{c} \approx 0.26$ to $z_{\text {ref }} / \delta_{c} \approx 0.09$ as the contour level is increased from figure $23(a)$ to $23(d)$. Figure 24 collates this information showing how the choice of $R_{u u}$ contour level (from which $z_{c \theta \text { (min) }}$ is determined) influences the location of the detachment point. The result is shown on outer scaled axes, with the width of the hatched region indicative of the uncertainty in determining the detachment point from outer-collapsed profiles such as those shown in figure 23. The detachment point is determined from the sudden gradient change noted to occur in $\partial z_{c \theta(\min )} / \partial z_{r e f}$, and is presented in terms of $z_{\text {ref }} / \delta_{c}$ (the point about which two-point correlations are performed). For any given contour level $R_{u u}=n$ and regardless of Reynolds number, a corresponding $z_{r e f} / \delta_{c}$ greater than the curve will indicate that the $n$ iso-correlation contour is in the detached regime, whilst with $z_{\text {ref }} / \delta_{c}$ below the curve, the same contour can be considered to be in an attached state.

Let us briefly consider the implications of the attached/detached correlation regimes, taking the $R_{u u}=0.4$ contour levels of figures 21 and 22 as a starting point. The proximity of this contour to the wall is actually a statistical measure of how well fluctuations at $z_{\text {ref }}$ are correlated with fluctuations near the wall. For the case shown 


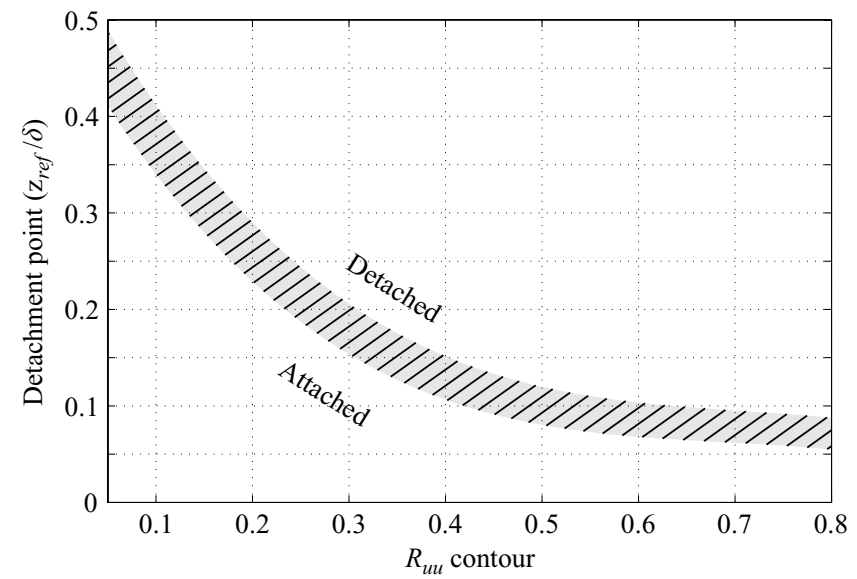

FIGURE 24. Variation of the 'detachment' point with $R_{u и}$ contour level for the $45^{\circ}$ case. The width of the hatched region represents the uncertainty in extracting this result. The detachment point is determined for each contour level from the rapid change in $\partial z_{c \theta(\min )} / \partial z_{\text {ref }}$ (see, for example, figure 23 ).

in figure $21\left(R_{u u}=0.4\right)$, events throughout the log region maintain a correlation of at least 0.4 with the near-wall region $\left(z^{+} \approx 40\right)$, seeming to signify a structure whose influence spans all the way down from $z_{\text {ref }}$ to the top of the viscous buffer region. Beyond this approximate limit, the rapid change in $\partial z_{c \theta(\min )} / \partial z_{\text {ref }}$ indicates that fluctuations at $z_{\text {ref }}$ are increasingly less likely to be correlated with those at the wall. This implies that beyond a certain height, structures may pinch-off from the wall, thus breaking the communicative link between $z_{\text {ref }}$ and the buffer region (leading to a sudden reduction in correlations). Figure 16(d) provides a circumstantial example of the type of instantaneous flow field that could account for this behaviour. For $z^{+} \lesssim 500$, alternating regions of positive and negative fluctuation extend almost fully down to the wall. Events at $z_{\text {ref }}$ have a direct and obvious connection with those at the wall. Hence, in this region we might expect to find the curtailed or splatted $R_{u u}$ contours that are typical of what we have termed the attached regime. Beyond this limit, we see evidence of a rapid jump in scales resulting in far larger regions of positive and negative fluctuation. Unlike the fluctuations below $z^{+} \approx 500$, these larger regions no longer appear to extend to the wall. This scenario would lead to an $R_{u u}$ correlation that attenuated some distance away from the wall (the lifted contours typified by the detached regime). Such $R_{u u}$ behaviour, and the jump in scales witnessed in figure $16(d)$ can be accommodated within the scenario of nested hairpin packets as suggested by Adrian et al. (2000b). In the sketch of their model, larger detached packets (packets comprised of structures that no longer appear to be trailing legs through the near-wall region) are shown over-running smaller near-wall structures. Certainly, a packet that had lifted fully away from the wall (and was 'skipping' over near-wall structures) could result in a correlation that no longer extended to the wall. When Perry \& Marusic (1995) extended the attached eddy hypothesis to model the wake region, they found it necessary to include a detached wake-type eddy that did not extend a velocity signature down towards the wall (in addition to the representative attached hairpin-type eddy) to accurately model the full extent of the turbulent boundary layer. The existence of such a structure can easily explain the detached correlations noted in this section.

Regardless of the finer details of the turbulent structure, figure 24 is a concise result, documenting the mean influence of outer-scale lifted events on the near-wall 
region. The cross-hatched curve implies that, on average, no event beyond the log region $\left(z / \delta_{c}>0.2\right)$ has a correlation with the buffer region that is greater than approximately 0.3 . It would be interesting to look at a similar curve resolved for an adverse pressure gradient turbulent boundary layer. In this case, the wake flow (and wake-type detached structures) will become increasingly dominant. This should manifest as an altered attached/detached regime. This would be consistent with a shrinking log-law region in an increasing adverse pressure gradient flow. In addition, any turbulence models (i.e. attached eddy hypothesis, LES) should be tested on their ability to reproduce this phenomena, without which this subtle detail of the turbulence structure is clearly missing or misrepresented.

\section{Conclusions}

From stereoscopic PIV measurements, on planes inclined both with and against the principle vorticity axis of a proposed hairpin model, we have been able to draw the following conclusions.

(i) With careful attention to experimental technique, it has been shown that inclined cross-stream PIV measurements can accurately capture the salient features of the turbulent boundary layer, over a limited yet significant range of Reynolds numbers.

(ii) Comparisons of in-plane swirling activity for the $45^{\circ}$ and $135^{\circ}$ planes support the existence of an inclined eddy structure. This inclined tendency is further evidenced by the linear stochastic estimate of the low-speed event, which shows a conditional counter-rotating vortex pair arranged about the low-speed event and inclined at approximately $20^{\circ}$ to the mean axis (at $z^{+} \approx 300$ ). In addition to this, the two-point correlations exhibit the hallmarks of an inclined structure, with correlations extending further in the $z^{\prime}$ direction for the $45^{\circ}$ plane than for the $135^{\circ}$ case (investigated in detail by Ganapathisubramani et al. 2005).

(iii) Conditional analysis on a given signed swirl event has revealed a weak predominance for a counter-rotating arrangement of vortex pairs (turning such that ejection occurs between the two vortices). Such arrangements are presupposed by the hairpin model. However, the weakness of this preference implies an underlying structural asymmetry. Whilst hairpin-type eddies may be a good representative structure in an average sense, the instantaneous form would appear to be closer to the asymmetric cane-type structures observed by (among others) Robinson (1991).

(iv) Instantaneously, we see evidence consistent with the occurrence of hairpin packets. Tall low-speed streaks are noted extending a considerable distance from the wall (often far beyond the edge of the log region). Such events are flanked by highspeed regions (visible in the two-point correlations, the LSE event and instantaneous flow-fields). In the $135^{\circ}$ plane it is noted that multiple patches of swirl often occupy the vertically aligned shear layers between these high- and low-speed regions. Such flow patterns are consistent with a $135^{\circ}$ slice through a representative hairpin packet.

(v) Two-point correlations, at a range of Reynolds numbers, have revealed that the dominant spanwise length scales (in $u, v$ and $w$ ) scale with boundary-layer thickness throughout the log and wake regions.

(vi) Below a given $z_{r e f}, R_{u u}$ contours can appear curtailed or splatted onto the wall. Beyond this $z_{\text {ref }}$, the same contours begin to 'lift' or 'separate' from the wall (initially noted at limited wall-normal stations by Ganapathisubramani et al. 2005). We have designated these two regimes as attached (events at $z_{\text {ref }}$ are still strongly influencing or influenced by the wall) and detached (a noticeable de-correlation between fluctuations at $z_{r e f}$ and those at the wall). Although this detachment point 
varies with contour level, the actual 'lift-off' occurs at a consistent $z_{\text {ref }} / \delta$ for any given contour (the detachment point scales with outer variables). As an example, the $R_{u u}=0.3$ contour decouples from the wall at $z_{\text {ref }} / \delta=0.2$ (the approximate edge of the log region) for all Reynolds numbers tested. These results are significant since they provide a Reynolds-number-independent measure of the feedback or influence between outer lifted events and the wall. Overall, it is proposed that these results indicate a general separation of hairpin-type structures (and hence reduction in wallinfluence or feedback) as they grow beyond the logarithmic region. This finding should be of interest to all those who seek to model turbulent boundary layers (and perhaps also to those who seek to control them). Also, since the decoupling of events seems to imply a degree of separation, it is proposed that this measure may also be well suited to capturing important structural changes that occur in non-zero pressure gradient turbulent boundary layers. See for example Perry \& Marusic (1995); Marusic \& Perry (1995) and also Chong et al. (1998) (who analysed structural changes in an adverse pressure gradient DNS boundary layer).

The authors gratefully acknowledge support from the National Science Foundation (Grant CTS-0324898) and the David and Lucile Packard Foundation. We are indebted to B. Ganapathisubramani for his help with post-processing and many useful discussions during the course of this study.

\section{REFERENCES}

ACARlar, M. S. \& SMith, C. R. 1987 a A study of hairpin vortices in a laminar boundary layer. Part 1. Hairpin vortices generated by a hemisphere protuberance. J. Fluid Mech. 175, 1-41.

AcARlar, M. S. \& Smith, C. R. $1987 b$ A study of hairpin vortices in a laminar boundary layer. Part 2. Hairpin vortices generated by fluid injection. J. Fluid Mech. 175, 43-83.

Adrian, R. J. 1991 Particle-imaging techniques for experimental fluid mechanics. Annu. Rev. Fluid Mech. 23, 261-304.

Adrian, R. J., Christensen, K. T. \& Lui, Z.-C. $2000 a$ Analysis and interpretation of instantaneous turbulent velocity fields. Exps. Fluids 29, 275-290.

Adrian, R. J., Meinhart, C. D. \& Tomkins, C. D. $2000 b$ Vortex organization in the outer region of the turbulent boundary layer. J. Fluid Mech. 422, 1-54.

Adrian, R. J. \& MoIN, P. 1988 Stochastic estimation of organized turbulent structure: homogeneous shear flow. J. Fluid Mech. 190, 531-559.

Adrian, R. J. \& SpalaRT, P. 1988 Stochastic estimation of organized turbulent structure: homogeneous shear flow. J. Fluid Mech. 190, 531-559.

Del Álamo, J. C. \& Jiménez, J. 2003 Spectra of the very large anisotropic scales in turbulent channels. Phys. Fluids 15, 41-44.

del Álamo, J. C., Jiménez, J., Zandonade, P. \& Moser, R. D. 2004 Scaling of the energy spectra of turbulent channels. J. Fluid Mech. 500, 135-144.

Balint, J.-L., Wallace, J. M. \& Vukoslavcevic, P. 1991 The velocity and vorticity vector fields of a turbulent boundary layer. Part 2. Statistical properties. J. Fluid Mech. 228, 53-86.

Chong, M. S., Soria, J., Perry, A. E., Chacin, J., Cantwell, B. J. \& Na, Y. 1998 Turbulence structures of wall-bounded shear flows found using DNS data. J. Fluid Mech. 357, 225-247.

Christensen, K. T. 2004 The influence of peak-locking errors on turbulence statistics computed from PIV ensembles. Exps. Fluids 36, 484-497.

Christensen, K. T. \& Adrian, R. J. 2001 Statistical evidence of hairpin vortex packets in wall turbulence. J. Fluid Mech. 431, 433-443.

Ganapathisubramani, B., Hutchins, N., Hambleton, W. T., Longmire, E. K. \& Marusic, I. 2005 Investigation of large-scale coherence in a turbulent boundary layer using two-point correlations. J. Fluid Mech. 524, 57-80.

Ganapathisubramani, B., Longmire, E. K. \& Marusic, I. 2003 Characteristics of vortex packets in turbulent boundary layers. J. Fluid Mech. 478, 35-46. 
Head, M. R. \& Bandyopadhyay, P. 1981 New aspects of turbulent boundary-layer structure. J. Fluid Mech. 107, 297-337.

Honkan, A. \& ANDreopoulos, Y. 1997 Vorticity, strain-rate and dissipation characteristics in the near-wall region of turbulent boundary layers. J. Fluid Mech. 350, 29-96.

Hutchins, N., Ganapathisubramani, B. \& Marusic, I. 2004 Dominant spanwise Fourier modes, and the existence of very large scale coherence in turbulent boundary layers. In Proc. Fifteenth Australasian Fluid Mech. Conf. (ed. M. Behnia, W. Lin \& G. D. McBain).

JimÉnez, J. \& DEL Álamo, J. C. 2004 Computing turbulent channels at experimental Reynolds numbers. In Proc. Fifteenth Australasian Fluid Mech. Conf. (ed. M. Behnia, W. Lin \& G. D. McBain).

Kim, K. C. \& Adrian, R. J. 1999 Very large scale motions in the outer layer. Phys. Fluids 11, $417-422$.

Kline, S. J., Reynolds, W. C., Schraub, F. A. \& Rundstadler, P. W. 1967 The structure of turbulent boundary layers. J. Fluid Mech. 30, 741-773.

KLINE, S. J. \& Robinson, S. K. 1988 Quasi-coherent structures in the turbulent boundary layer: Part 1. Status report on a community wide summary of the data. In Near-wall Turbulence (ed. S. J. Kline \& N. H. Afghan), pp. 200-217. Hemisphere.

Liu, Z., Adrian, R. J. \& Hanratty, T. J. 2001 Large-scale modes of turbulent channel flow: transport and structure. J. Fluid Mech. 448, 53-80.

MCLEAN, I. R. 1990 The near-wall eddy structure in an equilibrium turbulent boundary layer. $\mathrm{PhD}$ thesis, University of Southern California, USA.

Marusic, I. 2001 On the role of large-scale structures in wall turbulence. Phys. Fluids 13, 735-743.

Marusic, I. \& Perry, A. E. 1995 A wall wake model for the turbulent structure of boundary layers. Part 2. Further experimental support. J. Fluid Mech. 298, 389-407.

Morn, P. \& KIM, J. 1985 The structure of the vorticity field in turbulent channel flow. Part 1. Analysis of instantaneous fields and statistical correlations. J. Fluid Mech. 155, 441-464.

Na, Y., Hanratty, T. J. \& LiU, Z. 2001 The use of DNS to define stress producing events for turbulent flow over a smooth wall. Flow Turb. Combust. 66, 495-512.

Offen, G. R. \& KLINE, S. J. 1975 A proposed model of the bursting process in turbulent boundary layers. J. Fluid Mech. 70, 209-228.

Panton, R. L. 2001 Overview of the self-sustaining mechanisms of wall turbulence. Prog. Aerospace Sci. 37, 341-383.

Perry, A. E. \& Chong, M. S. 1982 On the mechanism of wall turbulence. J. Fluid Mech. 119, $173-217$.

Perry, A. E. \& ChOng, M. S. 1994 Topology of flow patterns in vortex motions and turbulence. Appl. Sci. Res. 53, 357-374.

Perry, A. E. \& Marusic, I. 1995 A wall wake model for the turbulent structure of boundary layers. Part 1. Extension of the attached eddy hypothesis. J. Fluid Mech. 298, 361-388.

Robinson, S. K. 1990 Kinematics of turbulent boundary layer structure. PhD thesis, Stanford University, Stanford, CA.

Robinson, S. K. 1991 Coherent motions in turbulent boundary layers. Annu. Rev. Fluid Mech. 23, 601-639.

SpalaRt, P. R. 1988 Direct numerical simulation of a turbulent boundary layer upto $R_{\theta}=1410$. J. Fluid Mech. 187, 61-98.

Theodorsen, T. 1952 Mechanism of turbulence. In Proc. Second Midwestern Conf. Fluid Mech. March 17-19. Ohio State Univesity, Columbus, Ohio.

Tomkins, C. D. \& AdRian, R. J. 2003 Spanwise structure and scale growth in turbulent boundary layers. J. Fluid Mech. 490, 37-74.

Townsend, A. A. 1956 The Structure of Turbulent Shear Flow. Cambridge University Press.

Wallace, J. M. 1985 The vortical structure of bounded turbulent shear flow. Lecture Notes in Physics, vol. 235, pp. 253-267. Springer.

Wark, C. E., Naguib, A. M. \& Robinson, S. K. Springer. 1991 Scaling of spanwise length scales in a turbulent boundary layer. AIAA Paper 91-0235, 29th Aerospace Sciences Meeting, Reno, Nevada.

Zhou, J., Adrian, R. J., Balachandar, S. \& Kendall, T. M. 1999 Mechanisms for generating coherent packets of hairpin vortices in channel flow. J. Fluid Mech. 387, 353-396. 


\section{University Library}

\section{- M M I N E R VA A gateway to Melbourne's research publications}

Minerva Access is the Institutional Repository of The University of Melbourne

\section{Author/s:}

Hutchins, N.;Hambleton, W. T.;MARUSIC, IVAN

Title:

Inclined cross-stream stereo particle image velocimetry measurements in turbulent boundary layers

Date:

2005

Citation:

Hutchins, N., Hambleton, W. T., \& Marusic, I. (2005). Inclined cross-stream stereo particle image velocimetry measurements in turbulent boundary layers. Journal of Fluid Mechanics, $541,21-54$.

Publication Status:

Published

Persistent Link:

http://hdl.handle.net/11343/34719 